\title{
Exponential Feedback Passivity of Switched Polynomial Nonlinear Systems
}

\author{
Huawei Zhu $\mathbb{D}^{1,2}$ and Xiaorong Hou (iD) \\ ${ }^{1}$ School of Energy Science and Engineering, University of Electronic Science and Technology of China, \\ Chengdu 611731, China \\ ${ }^{2}$ Dazhou Industrial Technology Institute of Intelligent Manufacturing, Sichuan University of Arts and Science, \\ Dazhou 635000, China \\ Correspondence should be addressed to Xiaorong Hou; houxr@uestc.edu.cn
}

Received 7 November 2017; Revised 26 February 2018; Accepted 7 March 2018; Published 13 May 2018

Academic Editor: Oleg V. Gendelman

Copyright (C) 2018 Huawei Zhu and Xiaorong Hou. This is an open access article distributed under the Creative Commons Attribution License, which permits unrestricted use, distribution, and reproduction in any medium, provided the original work is properly cited.

\begin{abstract}
The exponential feedback passivity problem of switched polynomial nonlinear systems is studied. To obtain this aim, a method of parameterization of controller is presented and parameter solution algorithm is described. Then, the addressed method is utilized to solve the robust stabilization for a class of switched polynomial nonlinear systems with parameter uncertainties and external disturbance. This result extends the previous results on exponential feedback passivity from the case of nonlinear systems to switched nonlinear systems. A numerical example is given to demonstrate the effectiveness of the proposed result.
\end{abstract}

\section{Introduction}

Switched polynomial nonlinear systems (SPNSs) [1-3] are a class of dynamical systems consisting of nonlinear subsystems with polynomial structure and a switching law, which defines a specific subsystem being activated during a certain interval of time. Due to the theoretical development as well as practical applications, analysis and synthesis of switched systems have recently gained considerable attention. Many tools, such as single Lyapunov function [4], multiple Lyapunov functions [5], and average dwell time [6], have been proved effective in solving the problem of stability and stabilization for switched nonlinear systems. In addition, in practical control systems, the parameter uncertainty and external disturbance are common, which implies that it is meaningful to study the robust stabilization of switched polynomial nonlinear system with parameter uncertainties and external disturbance [6-8].

On the other hand, as a particular case of dissipativity, passivity was introduced in [9] and later generalized in [10]. In virtue of the property of keeping a system internal stability, passivity theory is extensively used as an effective tool for the stability analysis and synthesis of nonlinear systems [11-22]. Recently, the exponential passivity problem for nonlinear systems has attracted the attention of many scholars. In [23], the concept of exponential passivity was introduced. In this result, it needs to satisfy that there exists an $r$-continuously differentiable storage function $V_{s}(\cdot)$ with supply rate $r(u, y)=u^{T} y$ meeting some conditions. Using exponentially weighted system storage functions with appropriate exponentially weighted supply rates, Chellaboina and Haddad [24] introduced the notion of exponential dissipativity, which was more general than the notion of exponential passivity introduced in [23]. In [25], a delaydependent exponential passivity condition for memristive neural networks was proposed in terms of linear matrix inequalities. By constructing a proper Lyapunov-Krasovskii functional, utilizing free-weighting matrix method and some stochastic analysis techniques, some delay-dependent sufficient conditions that ensure the exponential passivity of stochastic neural networks were deduced in [26]. In [27], by constructing an appropriate Lyapunov-Krasovskii functional 
and using the Wirtinger-based integral inequality to estimate its derivative, a delay-range-dependent and delay-ratedependent criterion was presented to ensure the augmented filtering dynamic system to be robustly exponentially passive with an expected dissipation. The exponential passivity of some systems can be ensured with the conditions of [2527]. Still and all, whether these conditions are true depend on the structure of the systems themselves. In addition, the nonlinear functions of the systems of [25-27] should satisfy the so-called Lipschitz condition, which restrict the application scope of these results. In [23], some feedback controllers were designed to obtain exponential passivity for a class of affine systems possessing globally defined normal form and factorisable high-frequency gain. In [28], a continuous output feedback controller was designed to solve the exponentially stability problem for a class of uncertain systems that contain a nominal part which is affine in the control and an uncertain part which is norm bounded by a known function. However, the results of $[23,28]$ are only suitable for some special systems. Based on the above discussions, we known that the related research on the exponential feedback passivity of nonlinear systems is still rather limited. Especially to the author's knowledge, there exist no results on the feedback exponential passivity of switched nonlinear systems so far, which is the motivation of the research of this paper.

In this paper, the exponential feedback passivity problem and the exponential passivity property are investigated for a class of SPNSs. The focus is on designing parameterized controller and solving out the feasible interval solution of the parameter. First, a concept of $C^{r}$-parameterized feedback exponentially passive of switched systems is introduced. Second, some parameterized symmetric matrix inequality conditions are proposed to ensure the exponential passivity of the SPNSs. Then, a parameter solution algorithm is formulated to solve the matrix inequalities. At last, the exponential passivity property is utilized to solve the robust stabilization problem of the SPNSs with parameter uncertainties and external disturbance.

The remainder of this paper is organized as follows. Section 2 is the problem formulation. The main contributions of this paper are then given in Section 3, in which a method of parameterization of controller is produced and a parameter solution algorithm is provided. In Section 4, a numerical example to support the results of this paper is given, which is followed by the conclusion in Section 5 .

Notations. The notations throughout this paper are quite standard. We use $P>0$ to denote a positive define matrix P. $\lambda_{M}(P)\left(\lambda_{m}(P)\right)$ means the largest (smallest) eigenvalue of matrix $P$. Let $\|P\|=\left(\sum_{j=1}^{n} \sum_{i=1}^{n} P(i, j)^{2}\right)^{1 / 2} \cdot L_{f(x)} V(x)$, $L_{g(x)} V(x)$ mean $(\partial V(x) / \partial x) f(x),(\partial V(x) / \partial x) g(x)$, respectively. $I$ denotes identity matrix with appropriate dimension. $R^{n}$ denotes the $n$ dimensional Euclidean space. Let $x \in R_{+}^{n}=$ $\left\{x \in R^{n}, x>0\right\} .\|x\|=\left(x^{T} x\right)^{1 / 2}$ refers to the Euclidean vector norm. $L_{2}[0,+\infty)$ is the space of square integrable vector over $[0,+\infty)$. " $P^{T \text { " }}$ denotes the transpose of the matrix $P$. " $P$-T" denotes the inverse of the matrix $P^{T}$. Matrices, if not explicitly stated, are assumed to have compatible dimensions.

\section{Problem Formulation and Preliminaries}

Consider following uncertain switched polynomial nonlinear system (SPNS) with the form

$$
\begin{aligned}
\dot{x}= & f_{\sigma(t)}(x)+\Delta f_{\sigma(t)}(t, x)+g_{\sigma(t)}(x) u_{\sigma(t)} \\
& +g_{\sigma(t) 1}(x) w(t), \quad f(0)=0 \\
y= & h_{\sigma(t)}(x),
\end{aligned}
$$

where $x \in R^{n}, y \in R^{p}, u \in R^{p}$, and $w \in R^{q}$ represent, respectively, the state variables, output, control, and external disturbance and $w(t) \in L_{2}[0,+\infty) . \sigma(t)$ : $[0, \infty) \rightarrow M=\left\{1,2, \ldots, m^{*}\right\}$, called switching signal, is a piecewise right continuous function. $m^{*}$ is a finite integer in the nonnegative integer set. $\sum=\left\{\left(t_{k}, i_{k}\right) \mid i_{k} \in M ; k=\right.$ $\left.0,1, \ldots ; 0=t_{0} \leq t_{1} \leq \cdots\right\}$ means the switching sequence corresponding to the switching signal $\sigma(t) . \sigma(t)=i, \quad(i \in M)$ means the $i$ th subsystem is active. For $\sigma(t)=i, f_{i}(x)$ and $h_{i}(x)$ are nonlinear polynomial functions, $\Delta f_{i}(x)$ express the parameter uncertainty, and $g_{i}(x)$ and $g_{i 1}(x)$ are polynomial matrices. Assume that the states of system (1) do not jump at switching instants.

Remark 1. For the SPNSs, all the terms of the matrices are polynomial functions about system states. The requirement of possessing polynomial structure is not very restrictive for system (1) since any continuous functions can be approximated by polynomials of sufficient large order.

In the following subsections, some definitions and lemma to be used in this paper are provided.

Definition 2 (see [29]). For a switching signal $\sigma(t)$ and any $T>T_{0} \geq 0$, If $N_{\sigma}\left(T, T_{0}\right) \leq N_{0}+\left(T-T_{0}\right) / \tau_{\alpha}, \tau_{\alpha}>0, N_{0} \geq 0$, $\tau_{\alpha}$ and $N_{0}$ are called, respectively, the average dwell time and the jitter bound, where $N_{\sigma}\left(T, T_{0}\right)$ is the switching numbers of $\sigma(t)$ over the interval $\left[T_{0}, T\right)$.

$N_{0}$ refers to the initial number of jitter. Without loss of generality, we make the value $N_{0}=0$.

Definition 3. Considering the following switched system:

$$
\begin{aligned}
& \dot{x}=f_{\sigma(t)}(x)+g_{\sigma(t)}(x) u_{\sigma(t)}, \\
& y=h_{\sigma(t)}(x),
\end{aligned}
$$

if, for $\forall i, j \in M$, any given average dwell time $\tau_{\alpha}$, there exist positive constant $\widehat{\alpha}_{i}, \mu \geq 1$, positive constants $a_{1}, a_{2}$, positive definite continuous function $V_{i}(x)$ called storage function with $V_{i}(0)=0$, and parameterized controller $u_{i}\left(x, k_{m}\right)=v_{i}\left(x, k_{m}\right)+r_{i}^{T} v_{i}(x)$ in which $v_{i}\left(x, k_{m}\right)$ is the part to parameterize the controller, $v_{i}(x)=-h_{i}(x)$ is the new input, $r_{i}$ is a weighting matrix with full column rank, $k_{m}$ is the parameter with serial number $m$, and there exists feasible interval solution for $k_{m}$, such that, $\forall t \geq 0$

(i)

$$
L_{f_{i}+g_{i} u_{i}} V_{i}(x) \leq v_{i}^{T} h_{i}-\widehat{\alpha}_{i} V_{i}(x)
$$


(ii)

$$
h_{i}(x)=r_{i} g_{i}^{T}(x) \frac{\partial V_{i}(x)}{\partial x}
$$

(iii)

$$
a_{1}\|x\|^{2} \leq V_{i}(x) \leq a_{2}\|x\|^{2}
$$

(iv)

$$
V_{i}(x) \leq \mu V_{j}(x)
$$

(v)

$$
\widehat{\alpha}_{i}>\frac{\ln (\mu)}{\tau_{\alpha}}
$$

hold. Then, under the parameterized controller $u_{i}\left(x, k_{m}\right)$ and switching law with average dwell time $\tau_{\alpha}$, the closedloop system (2) is said to be $C^{r}$-parameterized feedback exponentially passive.

Remark 4. In view of the switching law with average dwell time $\tau_{\alpha}$ and its related switching sequence $\sum$, letting $\widehat{\alpha}=$ $\min _{i \in M} \widehat{\alpha}_{i}$, for $t \in\left[t_{k}, t_{k+1}\right), k=0,1, \ldots$, by (3) and (6), similar to the process of (43), it can be obtained that

$$
\begin{aligned}
& V_{i_{k}}(x(t)) \leq \mu^{k} \exp \left(-\widehat{\alpha}\left(t-t_{0}\right)\right) V_{i_{0}}\left(x\left(t_{0}\right)\right) \\
& \quad+\int_{t_{k}}^{t} \exp (-\widehat{\alpha}(t-\tau)) v_{i_{k}}^{T}(\tau) y_{i_{k}}(\tau) d \tau \\
& \quad+\sum_{n=1}^{k} \int_{t_{n-1}}^{t_{n}}(\mu)^{k+1-n} \exp (-\widehat{\alpha}(t-\tau)) v_{i_{n-1}}^{T}(\tau) \\
& \cdot y_{i_{n-1}}(\tau) d \tau \leq \exp \left(-\widehat{\alpha}\left(t-t_{0}\right)+N_{\sigma}\left(t_{0}, t\right)\right. \\
& \quad \cdot \ln (\mu)) V_{i_{0}}\left(x\left(t_{0}\right)\right) \\
& \quad+\int_{t_{0}}^{t} \exp \left(-\widehat{\alpha}(t-\tau)+N_{\sigma}(\tau, t) \ln (\mu)\right) v^{T}(\tau) \\
& \quad y(\tau) d \tau .
\end{aligned}
$$

By Definition 2, it is obtained that $N_{\sigma}(\tau, t) \ln (\mu) \leq(t-$ $\tau)\left((\ln (\mu)) / \tau_{\alpha}\right)$, which implies that $-\widehat{\alpha}(t-\tau)+N_{\sigma}(\tau, t) \ln (\mu) \leq$ $\left((\ln (\mu)) / \tau_{\alpha}-\widehat{\alpha}\right)(t-\tau)$. Therefore,

$$
\begin{gathered}
V_{i_{k}}(x(t)) \leq \exp \left(-\widehat{\alpha}\left(t-t_{0}\right)+N_{\sigma}\left(t_{0}, t\right) \ln (\mu)\right) \\
\cdot V_{i_{0}}\left(x\left(t_{0}\right)\right)+\frac{1}{2} \exp \left(\left(\frac{\ln (\mu)}{\tau_{\alpha}}-\widehat{\alpha}\right) t\right) \\
\cdot \int_{t_{0}}^{t} 2 \exp \left(\left(\widehat{\alpha}-\frac{\ln (\mu)}{\tau_{\alpha}}\right) \tau\right) \nu^{T}(\tau) y(\tau) d \tau .
\end{gathered}
$$

In view of $V_{i_{k}}(x(t)) \geq 0$, by (7), when $x\left(t_{0}\right)=0$, it gives $\int_{t_{0}}^{t} e^{\varepsilon \tau} \widehat{r}(\nu(\tau), y(\tau)) d \tau \geq 0$, where $\varepsilon=\widehat{\alpha}-(\ln (\mu)) / \tau_{\alpha}>0$, $\widehat{r}(\nu(\tau), y(\tau))=2 \nu^{T}(\tau) y(\tau)$. Then, in view of Definitions 2.1 and 3.4 of [24], we know that system (2) is exponentially passive. Thus, the notion of $C^{r}$-parameterized feedback exponential passivity defined in Definition 3 in this paper is consistent with the notion of exponential passivity in [24].

Remark 5. The systems rendered to be $C^{r}$-exponentially passive by a parameterized feedback controller are called $C^{r}$ parameterized feedback exponentially passive systems or said to be feedback equivalent to $C^{r}$-parameterized exponentially passive systems. In Section 3, the $C^{r}$-parameterized feedback exponentially passive property will be used to solve the robust stabilization problem for the SPNSs with parameter uncertainties and external disturbance.

Lemma 6 (see [30]). If a vector function $f(x)$ with $f(0)=$ 0 has continuous $n$th order partial derivatives, $f(x)$ can be expressed as

$$
f(x)=a_{1}(x) x_{1}+\cdots+a_{n}(x) x_{n}=A(x) x,
$$

where $A(x)=\left[a_{1}(x), \ldots, a_{n}(x)\right], a_{i}(x)$ are vector functions, $i=1, \ldots, n, x=\left[x_{1}, \ldots, x_{n}\right]^{\mathrm{T}} \in R^{n}$. into

According to Lemma 6, system (1) can be transformed

$$
\begin{aligned}
\dot{x}= & A_{\sigma(t)}(x) x+\Delta f_{\sigma(t)}(t, x)+g_{\sigma(t)}(x) u_{\sigma(t)} \\
& +g_{\sigma(t) 1}(x) w(t),
\end{aligned}
$$

where $A_{\sigma(t)}(x)=\left[a_{\sigma(t) 1}(x), \ldots, a_{\sigma(t) n}(x)\right] \in R^{n \times n}$ is called structural matrix.

For $i \in M$, choose positive definite symmetric matrix $P_{i}$. In view of $P_{i}>0$, there must exist matrices $A_{i 1}(x)$ such that $A_{i 1}(x)=A_{i}(x) P_{i}^{-1}$ where $P_{i}^{-1}$ are the inverse of the matrices $P_{i}$. Then, $A_{i}(x)$ can be expressed as $A_{i}(x)=A_{i 1}(x) P_{i}$ and system (1) can be transformed into the following equivalent representation:

$$
\begin{aligned}
\dot{x}= & \left(J_{\sigma(t)}(x)-R_{\sigma(t)}(x)\right) P_{\sigma(t)} x+\Delta f_{\sigma(t)}(t, x) \\
& +g_{\sigma(t)}(x) u_{\sigma(t)}+g_{\sigma(t) 1}(x) w(t),
\end{aligned}
$$

where $J_{i}(x)=(1 / 2)\left(A_{i}(x) P_{i}^{-1}-P_{i}^{-T} A_{i}^{T}(x)\right)$ is skewsymmetric and $R_{i}(x)=-(1 / 2)\left(A_{i}(x) P_{i}^{-1}+P_{i}^{-T} A_{i}^{T}(x)\right)$ is symmetric.

Remark 7. When $R_{i}(x) \geq 0$, the $i$ th subsystem described by (12) is called dissipated Hamiltonian system and $R_{i}(x)$ is the so-called dissipative matrix [11].

\section{Main Results}

In this section, the exponential feedback passivity of SPNSs is addressed with the method of parameterization of controller. In what follows, they will be accomplished in two subsections.

\subsection{Exponential Feedback Passivity}

Theorem 8. Under the switching law with the given average dwell time $\tau_{\alpha}$, the closed-loop system (1) without parameter 
uncertainties and external disturbance is globally exponentially stable, if it is said to be feedback equivalent to $C^{r}$-parameterized exponentially passive system as Definition 3.

Proof. Choose the following piecewise Lyapunov function:

$$
V(x(t))=V_{\sigma(t)}(x(t)) .
$$

Then, taking no regard of parameter uncertainties and letting $w=0$, the differential of $V_{i}(x)$ along the trajectory of the close-loop system (1) is obtained

$$
\dot{V}_{i}(x)=\frac{\partial V_{i}(x)}{\partial x} \dot{x}=\frac{\partial V_{i}(x)}{\partial x}\left[f_{i}(x)+g_{i}(x) u_{i}\right] .
$$

According to the switching law with average dwell time $\tau_{\alpha}$, the switching sequence $\sum=\left(t_{k}, i_{k}\right)$ is achieved. For $t \in$ $\left[t_{k}, t_{k+1}\right), k=0,1, \ldots$, noting that $v_{i}(x)=-h_{i}(x)$, by (3), (4), it is obtained that

$$
\dot{V}_{i_{k}}(x) \leq-\alpha_{i_{k}} V_{i_{k}}(x)-h_{i_{k}}^{T} h_{i_{k}} \leq-\widehat{\alpha} V_{i_{k}}(x),
$$

where $\widehat{\alpha}=\min _{i \in M} \alpha_{i}$.

Integrating both sides of (15) over $t \in\left[t_{k}, t_{k+1}\right), \forall k=$ $0,1,2, \ldots$ leads to

$$
V_{i_{k}}(x(t)) \leq \exp \left(-\widehat{\alpha}\left(t-t_{k}\right)\right) V_{i_{k}}\left(x\left(t_{k}\right)\right) .
$$

Then, according to the switching sequence $\sum$ and Definition 2, by (6), it follows from (16) that

$$
\begin{aligned}
& V_{i_{k}}(x(t)) \leq \mu^{k} \exp \left(-\widehat{\alpha}\left(t-t_{0}\right)\right) V_{i_{0}}\left(x\left(t_{0}\right)\right) \\
& \quad \leq \exp \left(\left(N_{0}+\frac{t-t_{0}}{\tau_{\alpha}}\right) \ln (\mu)-\widehat{\alpha}\left(t-t_{0}\right)\right) \\
& \cdot V_{i_{0}}\left(x\left(t_{0}\right)\right) .
\end{aligned}
$$

On the other hand,

$$
\begin{aligned}
& \left(N_{0}+\frac{t-t_{0}}{\tau_{\alpha}}\right) \ln (\mu)-\widehat{\alpha}\left(t-t_{0}\right) \\
& =N_{0} \ln (\mu)+\left[\frac{\ln (\mu)}{\tau_{a}}-\widehat{\alpha}\right]\left(t-t_{0}\right) \\
& =N_{0} \ln (\mu)-\widehat{\alpha}_{0}\left(t-t_{0}\right),
\end{aligned}
$$

where

$$
\widehat{\alpha}_{0}=\widehat{\alpha}-\frac{\ln (\mu)}{\tau_{\alpha}} .
$$

Substituting (18) into (17) yields

$$
V_{i_{k}}(x(t)) \leq \mu^{N_{0}} \exp \left(-\widehat{\alpha}_{0}\left(t-t_{0}\right)\right) V_{i_{0}}\left(x\left(t_{0}\right)\right) .
$$

According to (7) and noting that $\widehat{\alpha}=\min _{i \in M} \alpha_{i}$, it gives $\hat{\alpha}_{0}>0$. Furthermore, by (5), we have $V_{i_{k}}(x(t)) \geq$ $a_{1}\|x(t)\|^{2}, V_{i_{0}}\left(x\left(t_{0}\right)\right) \leq a_{2}\left\|x\left(t_{0}\right)\right\|^{2}$. Then, it follows from (20) that

$$
\|x(t)\| \leq \sqrt{\frac{a_{2}}{a_{1}} \mu^{N_{0}}} \exp \left(-\frac{1}{2} \widehat{\alpha}_{0}\left(t-t_{0}\right)\right)\left\|x\left(t_{0}\right)\right\|
$$

which implies the globally exponential stability of the closedloop system (1) without parameter uncertainties and external disturbance. This completes the proof.

Theorem 9. Considering system (1) without parameter uncertainty and external disturbance, for any given average dwell time $T_{\alpha}, i \in M$, if there exist positive definite symmetric matrices $P_{i}$, positive definite functions $V_{i}(x)=(1 / 2) x^{T} P_{i} x$, symmetrical polynomial parameter matrices $K_{i}\left(x, k_{m}\right)$, and positive constants $\widetilde{\alpha}_{i}$, system (1) can be transformed into the form of (12) and, for $\forall x \in R_{+}^{n}$,

$$
\begin{aligned}
G_{i} & =\frac{\partial V_{i}(x)}{\partial x}\left(R_{i}(x)\right. \\
& \left.+g_{i}(x) K_{i}\left(x, k_{m}\right) g_{i}^{T}(x)\right) \frac{\partial V_{i}^{T}(x)}{\partial x} \\
& -\widetilde{\alpha}_{i} V_{i}(x)>0 \\
h_{i}(x) & =r_{i}(x) g_{i}^{T}(x) P_{i} x \\
\widetilde{\alpha}_{i} & >\frac{\ln (\mu)}{T_{\alpha}}
\end{aligned}
$$

hold.

Then, under the switching law with average dwell time $T_{\alpha}$ and following parameterized controller

$$
\begin{aligned}
u_{i}\left(k_{m}, x\right) & =-\left[K_{i}\left(x, k_{m}\right)+r_{i}^{T}(x) r_{i}(x)\right] g_{i}^{T}(x) P_{i} x \\
& =v_{i}\left(x, k_{m}\right)+r_{i}^{T} v_{i}(x),
\end{aligned}
$$

system (1) is said to be feedback equivalent to $C^{r}$-parameterized exponentially passive systems.

Proof. Let $a_{1}=(1 / 2) \min _{i \in M} \lambda_{m}\left(P_{i}\right), a_{2}=(1 /$ 2) $\max _{i \in M} \lambda_{M}\left(P_{i}\right), a_{3}=\max _{i \in M}\left\|P_{i}\right\|, \mu=\max _{k, l \in M}\left(\lambda_{M}\left(P_{k}\right) /\right.$ $\left.\lambda_{m}\left(P_{l}\right)\right)$. Obviously, (5) and (6) hold.

Considering the $i$ th subsystem, according to the conditions $f_{i}(0)=0$ and Lemma 6 , system (1) can be transformed into the form of (12).

Choose the storage functions for each subsystem

$$
V_{i}(x(t))=\frac{1}{2} x^{T} P_{i} x \geq 0, \quad i \in M .
$$

In view of (22), noting that $v_{i}(x)=-h_{i}(x)$, the differential of $V_{i}(x)$ along the trajectory of the system (1) is obtained

$$
\begin{aligned}
& \dot{V}_{i}(x(t))=-\frac{\partial V_{i}(x)}{\partial x}\left(R_{i}(x) \frac{\partial V_{i}^{T}(x)}{\partial x}\right. \\
& \left.-g_{i}(x)\left(v_{i}\left(x, k_{m}\right)+r_{i}^{T} v_{i}(x)\right)\right) \\
& =-\frac{\partial V_{i}(x)}{\partial x}\left(R_{i}(x)\right. \\
& \left.+g_{i}(x) K_{i}\left(x, k_{m}\right) g_{i}^{T}(x)\right) \frac{\partial V_{i}^{T}(x)}{\partial x} \\
& +v_{i}^{T}(x) h_{i}(x) \leq-\widetilde{\alpha}_{i} V_{i}(x)+v_{i}^{T}(x) h_{i}(x)
\end{aligned}
$$

which means (3) holds. 
By (23) and (24), we know that (4) and (7) hold. According to Definition 3, the closed-loop system (1) without parameter uncertainty and external disturbance is said to be $C^{r}$-parameterized exponentially passive. This completes the proof.

Remark 10. In order to render that (3) holds, condition (22) should be satisfied. However, the major difficulty is to solve out the interval value of $k_{m}$ such that (22) holds over the entire system state space. According to Remark 1, expression $G_{i}$ in the left side of condition (22) is polynomial function. By taking advantage of this feature, a symbolic computation based algorithm is produced to solve the interval value of $k_{m}$ and $\widetilde{\alpha}_{i}$ in Section 3.2.

Remark 11. The aims of the parameterization of controller include two aspects. Firstly, compensate for $R_{i}$ and render it to be dissipative matrix: that is, $R_{i}(x)+g_{i}(x) K_{i}\left(x, k_{m}\right) g_{i}^{T}(x) \geq 0$. Secondly, render that the storage function $V_{i}(x)$ has decay rate $\widetilde{\alpha}_{i}$ along each subsystem. In Theorem 13 and Remark 14, we will know that the robust stabilization problem of system (1) can also be solved if the values of $\widetilde{\alpha}_{i}$ are big enough. As a matter of fact, by adjusting the values of $k_{m}$ contained in the parameterized controller (25), the values of $\widetilde{\alpha}_{i}$ can be improved according to their feasible interval solutions.

In the following subsection, the $C^{r}$-parameterized feedback exponential passivity property of system (1) is utilized to solve its robust stabilization problem. Firstly, introduce an assumption as follows.

Assumption 12 (see [22]). Considering the uncertain terms of system (1), there exists nonnegative function $\varsigma(t)$, which satisfies $\int_{t_{0}}^{t} \varsigma(\tau) d \tau \leq \kappa\left(t-t_{0}\right)+\eta$ for some nonnegative constants $\kappa, \eta$, such that $\left\|\Delta f_{i}(t, x)\right\| \leq \varsigma(t)\|x\|, \forall i \in M$ hold for all $x$.

The uncertain term $\Delta f_{i}(t, x)$ described by Assumption 12 is usual in nonlinear system [22].

In this paper, the robust stabilization problem of system (1) is summarized as follows: for a given disturbance attenuation level $\gamma>0$, given average dwell time $\tau_{\alpha}$, parameterized controllers $u_{i}\left(k_{m}, x\right), i \in M$ in which $k_{m}$ are the parameters with serial number $m$ and there exist feasible interval solutions for $k_{m}$, such that

(a) the closed-loop system (1) is said to be globally robustly exponentially stable when $w=0$,

(b) the closed-loop system (1) has $L_{2}$ gain from $w$ to $y$ for all admissible uncertainties. That is to say, there exists a constant $\widetilde{\lambda} \geq 0$ and a real-valued function $\widetilde{v}(x)$ with $\widetilde{v}(0)=0$, such that

$$
\begin{gathered}
\int_{t_{0}}^{t} \exp \left(-\tilde{\lambda}\left(t-t_{0}\right)\right) y^{T}(t) y(t) d \tau \\
\leq \int_{t_{0}}^{t} \gamma^{2} w^{T} w d \tau+\widetilde{v}\left(x\left(t_{0}\right)\right)
\end{gathered}
$$

holds.
Theorem 13. Suppose the conditions of Theorem 9 are satisfied and the uncertain terms of system (1) satisfy Assumption 12. For any given disturbance attenuation level $\gamma$, if there exist positive constants $\alpha_{i}, i \in M, a_{3}$ such that (29), (30), and (31) hold,

$$
\begin{aligned}
& \widetilde{\alpha}_{i} V_{i}(x)-\frac{1}{4 \gamma^{2}} \frac{\partial V_{i}(x)}{\partial x} g_{i 1}(x) g_{i 1}^{T}(x) \frac{\partial V_{i}^{T}(x)}{\partial x} \\
& >\alpha_{i} V_{i}(x), \\
& \left\|\frac{\partial V_{i}(x)}{\partial x}\right\| \leq a_{3}\|x\|, \\
& \alpha_{i}-\frac{\ln \left(\mu \exp \left(a_{3} \eta / a_{1}\right)\right)}{\tau_{\alpha}}-\frac{a_{3}}{a_{1}} \kappa>0,
\end{aligned}
$$

where $\widetilde{\alpha}_{i}, a_{1}, \mu$, and $V_{i}(x)$ are the same as Theorem 9 and $\tau_{\alpha}$ is a reassigned average dwell time. Then, the robust stabilization problem is solvable for system (1) under the parameterized controller (25) and switching law with average dwell time $\tau_{\alpha}$.

Proof. Choose the storage functions $V_{i}(x(t))=(1 / 2) x^{T} P_{i} x \geq$ $0, i \in M$ as (26). By the switching law with average dwell time $\tau_{\alpha}$, the switching sequence $\sum$ is achieved. According to Theorem 9, the system (1) without parameter uncertainty and external disturbance is said to be $C^{r}$-parameterized exponentially passive. Then, by Definition 3 and (29), for $t \in\left[t_{k}, t_{k+1}\right), k=0,1, \ldots$, it is obtained that

$$
\begin{aligned}
\dot{V}_{i_{k}}(x(t)) \leq & -\alpha_{i_{k}} V_{i_{k}}(x)-h_{i_{k}}^{T}(x) h_{i_{k}}(x) \\
& -\frac{1}{4 \gamma^{2}} \frac{\partial V_{i_{k}}(x)}{\partial x} g_{i_{k} 1}(x) g_{i_{k} 1}^{T}(x) \frac{\partial V_{i_{k}}^{T}(x)}{\partial x} \\
& +\frac{\partial V_{i_{k}}(x)}{\partial x} \Delta f_{i_{k}}(t, x) \\
& +\frac{\partial V_{i_{k}}(x)}{\partial x} g_{i_{k} 1}(x) w .
\end{aligned}
$$

In addition, combining with Assumption 12, (5) and (30) lead to

$$
\begin{aligned}
\frac{\partial V_{i_{k}}(x)}{\partial x} \Delta f_{i_{k}}(t, x) & \leq \frac{\partial V_{i_{k}}(x)}{\partial x} \varsigma(t)\|x\| \\
& \leq a_{3}\|x\| \varsigma(t)\|x\| \leq \frac{a_{3}}{a_{1}} \varsigma(t) V_{i_{k}}(x) .
\end{aligned}
$$
that

When $w \equiv 0$, it is obtained by substituting (33) into (32)

$$
\begin{aligned}
\dot{V}_{i_{k}}(x(t)) & \leq-\left(\alpha_{i_{k}}-\frac{a_{3}}{a_{1}} \varsigma(t)\right) V_{i_{k}}(x)-h_{i_{k}}^{T}(x) h_{i_{k}}(x) \\
& \leq-\left(\alpha_{i_{k}}-\frac{a_{3}}{a_{1}} \varsigma(t)\right) V_{i_{k}}(x) \\
& \leq-\left(\alpha-\frac{a_{3}}{a_{1}} \varsigma(t)\right) V_{i_{k}}(x),
\end{aligned}
$$

where $=\min _{i \in M} \alpha_{i}$. 
Let $\xi=\exp \left(a_{3} \eta / a_{1}\right), \widetilde{\alpha}=\alpha-\left(a_{3} / a_{1}\right) \kappa, \Phi_{i_{k}}(t, \tau)=$ $\xi \exp (-\widetilde{\alpha}(t-\tau))$. Integrating both sides of (34) over $t \in$ $\left[t_{k}, t_{k+1}\right), \forall k=0,1,2, \ldots$ leads to

$$
\begin{gathered}
V_{i_{k}}(x(t)) \leq\left[\exp \left(-\alpha\left(t-t_{k}\right)\right)+\frac{a_{3}}{a_{1}} \int_{t_{k}}^{t} \varsigma(\tau) d \tau\right] \\
\cdot V_{i_{k}}\left(x\left(t_{k}\right)\right) \\
\leq\left[\exp \left(-\alpha\left(t-t_{k}\right)\right)+\frac{a_{3}}{a_{1}} \kappa\left(t-t_{k}\right)+\frac{a_{3}}{a_{1}} \eta\right] \\
\cdot V_{i_{k}}\left(x\left(t_{k}\right)\right)=\Phi_{i_{k}}\left(t, t_{k}\right) V_{i_{k}}\left(x\left(t_{k}\right)\right) .
\end{gathered}
$$

Obviously, according to the structural characteristic of $\Phi_{i_{k}}\left(t, t_{k}\right)$, switching sequence $\sum$, Definition 2 , (6), over the time interval $\left[t_{0}, t\right]$, it follows from (35) that

$$
\begin{aligned}
& V(x(t)) \leq \Phi_{i_{k}}\left(t, t_{k}\right) \mu \Phi_{i_{k-1}}\left(t_{i_{k}}, t_{i_{k-1}}\right) \cdots \mu \Phi_{i_{0}}\left(t_{i_{1}}, t_{i_{0}}\right) \\
& \cdot V\left(x\left(t_{0}\right)\right) \leq \mu^{k} \xi^{k+1} \exp \left(-\widetilde{\alpha}\left(t-t_{0}\right)\right) V_{i_{0}}\left(x\left(t_{0}\right)\right) \\
& \leq \xi \exp \left(\left(N_{0}+\frac{t-t_{0}}{\tau_{\alpha}}\right) \ln (\mu \xi)-\widetilde{\alpha}\left(t-t_{0}\right)\right) \\
& \cdot V_{i_{0}}\left(x\left(t_{0}\right)\right) .
\end{aligned}
$$

Besides, similar to (18), considering Definition 2, it is obtained that

$$
\begin{aligned}
& \left(N_{0}+\frac{t-t_{0}}{\tau_{\alpha}}\right) \ln (\mu \xi)-\tilde{\alpha}\left(t-t_{0}\right) \\
& =N_{0} \ln (\mu \xi)+\left[\frac{\ln (\mu \xi)}{\tau_{a}}-\tilde{\alpha}\right]\left(t-t_{0}\right) \\
& =N_{0} \ln (\mu \xi)-\bar{\alpha}_{0}\left(t-t_{0}\right),
\end{aligned}
$$

where

$$
\bar{\alpha}_{0}=\alpha-\frac{\ln (\mu \xi)}{\tau_{\alpha}}-\frac{a_{3}}{a_{1}} \kappa
$$

Substituting (37) into (36) yields

$$
V_{i_{k}}(x(t)) \leq \mu^{N_{0}} \xi^{N_{0}+1} \exp \left(-\bar{\alpha}_{0}\left(t-t_{0}\right)\right) V_{i_{0}}\left(x\left(t_{0}\right)\right) .
$$

Noting that $\alpha=\min _{i \in M} \alpha_{i}$, due to (31), it gives $\bar{\alpha}_{0}>0$. Furthermore, by (5), we have $V(x(t)) \geq \alpha_{1}\|x(t)\|^{2}, V\left(x\left(t_{0}\right)\right) \leq$ $\alpha_{2}\left\|x\left(t_{0}\right)\right\|^{2}$. Then, it follows from (39) that

$$
\|x(t)\| \leq \sqrt{\frac{a_{2}}{a_{1}} \mu^{N_{0}} \xi^{N_{0}+1}} \exp \left(-\frac{1}{2} \bar{\alpha}_{0}\left(t-t_{0}\right)\right)\left\|x\left(t_{0}\right)\right\|
$$

which implies the globally exponential stability of the closedloop system.
Next, the $L_{2}$ gain disturbance attenuation problem of system (1) from $w$ to $y$ is studied. With the similar procedure of (34), for $t \in\left[t_{k}, t_{k+1}\right), k=0,1, \ldots$, by (32) and (33), it gives

$$
\begin{aligned}
\dot{V}_{i_{k}}(x(t)) \leq & -\left(\alpha_{i_{k}}-\frac{a_{3}}{a_{1}} \varsigma(t)\right) V_{i_{k}}(x)-h_{i_{k}}^{T}(x) h_{i_{k}}(x) \\
& -\frac{1}{4 \gamma^{2}} x^{T} P_{i_{k}}^{T} g_{i_{k} 1}(x) g_{i_{k} 1}^{T}(x) P_{i_{k}} x \\
& +\frac{\partial V_{i_{k}}(x)}{\partial x} g_{i_{k} 1}(x) w \\
\leq & -\left(\alpha_{i_{k}}-\frac{a_{3}}{a_{1}} \varsigma(t)\right) V_{i_{k}}(x)-h_{i_{k}}^{T}(x) h_{i_{k}}(x) \\
& +\gamma^{2}\|w\|^{2} \\
\leq & -\left(\alpha-\frac{a_{3}}{a_{1}} \varsigma(t)\right) V_{i_{k}}(x)-h_{i_{k}}^{T}(x) h_{i_{k}}(x) \\
& +\gamma^{2}\|w\|^{2} .
\end{aligned}
$$

Let $\mathbb{Q}_{i_{k}}(t)=\gamma^{2} w^{2}(t) w(t)-h_{i_{k}}^{T}(x(t)) h_{i_{k}}(x(t))$. Then, integrating both sides of (41) over $t \in\left[t_{k}, t_{k+1}\right), \forall k=$ $0,1,2, \ldots$ yields

$$
\begin{aligned}
V_{i_{k}}(x(t)) \leq & \Phi_{i_{k}}\left(x\left(t, t_{k}\right)\right) V_{i_{k}}\left(x\left(t_{k}\right)\right) \\
& +\int_{t_{k}}^{t} \Phi_{i_{k}}(-(t, \tau)) \mathbb{Q}_{i_{k}}(\tau) d \tau .
\end{aligned}
$$

On the other hand, in view of the switching sequence $\sum=\left\{\left(t_{k}, i_{k}\right) \mid i_{k} \in M\right\}, \forall k=1,2, \ldots$, for $n \in\{1, \ldots, k\}$, $t \in\left[t_{k}, t_{k+1}\right), \tau \in\left[t_{n-1}, t_{n}\right)$, by Definition 2, it is obtained that $N_{\sigma}(\tau, t)=N_{\sigma}(n-1, t)=k+1-n$. Then, noting that $V_{i_{k}}(x(t)) \geq 0$, over the time interval $\left[t_{0}, t\right]$, it is obtained that

$$
\begin{aligned}
0 \leq & V_{i_{k}}(x(t)) \leq \mu^{k} \xi^{k+1} \exp \left(-\tilde{\alpha}\left(t-t_{0}\right)\right) V_{i_{0}}\left(x\left(t_{0}\right)\right) \\
& +\int_{t_{k}}^{t} \Phi_{i_{k}}(-(t, \tau)) \mathbb{Q}_{i_{k}}(\tau) d \tau \\
& +\xi \sum_{n=1}^{k} \int_{t_{n-1}}^{t_{n}}(\mu \xi)^{k+1-n} \exp (-\widetilde{\alpha}(t-\tau)) \mathbb{Q}_{i_{n-1}}(\tau) d \tau \\
& \leq \xi \exp \left(-\widetilde{\alpha}\left(t-t_{0}\right)+N_{\sigma}\left(t_{0}, t\right) \ln (\mu \xi)\right) \\
& \cdot V_{i_{0}}\left(x\left(t_{0}\right)\right) \\
& +\xi \int_{t_{0}}^{t} \exp \left(-\tilde{\alpha}(t-\tau)+N_{\sigma}(\tau, t) \ln (\mu \xi)\right) \\
& \cdot \mathbb{Q}(\tau) d \tau
\end{aligned}
$$

where $\mathbb{Q}(t)=\gamma^{2} w^{2}(t) w(t)-y^{T}(x(t)) y(x(t))$. 
Noticing that $\xi>0$, multiplying $\xi^{-1} \exp \left(-N_{\sigma\left(t_{0}, t\right)} \ln (\mu \xi)\right)$ with the two sides of (43) leads to

$$
\begin{aligned}
0 \leq & \exp \left[-\tilde{\alpha}\left(t-t_{0}\right)\right] V_{i_{0}}\left(x\left(t_{0}\right)\right)+\int_{t_{0}}^{t} \exp [-\tilde{\alpha}(t-\tau) \\
& \left.+\left(N_{\sigma}(\tau, t)-N_{\sigma}\left(t_{0}, t\right)\right) \ln (\mu \xi)\right] \mathbb{Q}(\tau) d \tau \\
& =\exp \left[-\tilde{\alpha}\left(t-t_{0}\right)\right] V_{i_{0}}\left(x\left(t_{0}\right)\right)+\int_{t_{0}}^{t} \exp [-\tilde{\alpha}(t \\
& \left.-\tau)-\frac{\left(\tau-t_{0}\right)}{\tau_{\alpha}} \ln (\mu \xi)\right] \mathbb{Q}(\tau) d \tau=\exp [-(\tilde{\alpha} \\
& \left.\left.+\frac{\ln (\mu \xi)}{\tau_{\alpha}}\right)\left(t-t_{0}\right)\right] V_{i_{0}}\left(x\left(t_{0}\right)\right)+\int_{t_{0}}^{t} \exp [-(\tilde{\alpha} \\
& \left.\left.+\frac{\ln (\mu \xi)}{\tau_{\alpha}}\right)(t-\tau)-\frac{\left(\tau-t_{0}\right)}{\tau_{\alpha}} \ln (\mu \xi)\right] \mathbb{Q}(\tau) d \tau,
\end{aligned}
$$

which implies that

$$
\begin{aligned}
& \int_{t_{0}}^{t} \exp \left[-\left(\tilde{\alpha}+\frac{\ln (\mu \xi)}{\tau_{\alpha}}\right)(t-\tau)-\frac{\left(\tau-t_{0}\right)}{\tau_{\alpha}} \ln (\mu \xi)\right] \\
& \cdot y^{T}(\tau) y(\tau) d \tau \leq \exp \left[-\left(\tilde{\alpha}+\frac{\ln (\mu \xi)}{\tau_{\alpha}}\right)(t\right. \\
& \left.\left.-t_{0}\right)\right] V_{i_{0}}\left(x\left(t_{0}\right)\right) \\
& +\int_{t_{0}}^{t} \exp \left(-\left(\tilde{\alpha}+\frac{\ln (\mu \xi)}{\tau_{\alpha}}\right)(t-\tau)\right) \\
& \cdot \exp \left(-\frac{\left(\tau-t_{0}\right)}{\left.\tau_{\alpha} \ln (\mu \xi)\right) \gamma^{2} w^{T}(\tau) w(\tau) d \tau}\right. \\
& \quad \leq \exp \left[-\left(\tilde{\alpha}+\frac{\ln (\mu \xi)}{\left.\tau_{\alpha}\right)}\left(t-t_{0}\right)\right] V_{i_{0}}\left(x\left(t_{0}\right)\right)\right. \\
& +\int_{t_{0}}^{t} \exp \left[-\left(\tilde{\alpha}+\frac{\ln (\mu \xi)}{\tau_{\alpha}}\right)(t-\tau)\right] \gamma^{2} w^{T}(\tau) \\
& +w(\tau) d \tau .
\end{aligned}
$$

Due to the positive properties of $a_{1}, a_{3}, \eta$, it gives $\xi>1$. In addition, by $\mu>1, \tilde{\alpha}>0$, and $\tau_{\alpha}>0$, it is easy to know that $\tilde{\alpha}+(\ln (\mu \xi)) / \tau_{\alpha}>0$. Then, for $t \rightarrow \infty$, it follows from (45) that

$$
\begin{aligned}
& \int_{t_{0}}^{+\infty} \exp \left[-\frac{\ln (\mu \xi)}{\tau_{\alpha}}\left(\tau-t_{0}\right)\right] y^{T}(\tau) y(\tau) \\
& \quad-\gamma^{2} w^{T}(\tau) w(\tau) d \tau \leq V\left(t_{0}\right)
\end{aligned}
$$

which means that the switched system achieves the $L_{2}$ gain level $\gamma$ from $w$ to $y$. This completes the proof.

Remark 14. Aside from the two aims described in Remark 11, the procedure of parameterization of controller has another effect that compensates some nonlinear actions for the external disturbance terms $g_{i 1}(x) w$. Hence, if the external disturbance structure matrices $g_{i 1}(x)$ contain state variables, the intervals of the parameters should be solved again by combining conditions (22) and (29). Namely, synthesize conditions (22) and (29) and obtain a new condition

$$
\begin{aligned}
G_{i} & =\frac{\partial V_{i}(x)}{\partial x}\left(R_{i}(x)+g_{i}(x) K_{i}\left(x, k_{m}\right) g_{i}^{T}(x)\right. \\
& \left.-\frac{1}{4 \gamma^{2}} g_{i 1}(x) g_{i 1}^{T}(x)\right) \frac{\partial V_{i}^{T}(x)}{\partial x}-\alpha_{i} V_{i}(x) \\
& \geq 0 .
\end{aligned}
$$

Solve conditions (31) and (47) with the PS algorithm described in Section 3.2 and obtain the interval values of the parameters $k_{m}$ and $\alpha_{i}$. Then the controller satisfying the requirements can be obtained as the same way of Theorem 9 .

In addition, it is easy to know that whether conditions (29) and (31) hold depends on the values of $\widetilde{\alpha}_{i}$. Considering this property, we have the following three ways to confirm the values of the parameters when the external disturbance structure matrices $g_{i 1}(x)$ do not contain state variables.

(1) If (29) and (31) are still established with the parameter values which are obtained according to Theorem 9, the controllers obtained in Theorem 9 are still effective in Theorem 13.

(2) If (29) or (31) is not established with the parameter values which is obtained according to Theorem 9, reselect the parameter values according to the interval value of the parameter (see Remark 10) obtained in Theorem 9.

(3) Resolve the interval values of the parameters according to (31) and (47) and reselect the parameter values. This way is always effective.

Remark 15. By the interval value of the parameter $k_{m}$, several specific values of $k_{m}$ can be chosen and several controllers are achieved. Then, the optimized controller can be got by comparing with the performance of system (1) under the above obtained different controllers. Considering that the dissipation of the system is closely related to some performances of the system [11], it is reasonable to optimize the system with this way (see Remark 11). In Section 4, the disturbance attenuation capability of the system is improved with this method.

3.2. Parameter Solution Algorithm. In order to obtain the interval values of the parameters restricted by (22), (24) or (31), (47), a symbolic computation based parameter solution (PS) algorithm is produced in this subsection. First, design the parameter matrices and obtain the parameterized controllers; secondly, according to conditions (22), (24) or (31), (47), obtain equivalent inequality set; thirdly, decompose in the parameter space and search out the required cell. At last, choose several values for each $k_{m}$ and obtain the optimized controller. In this subsection, we will just consider the case of (31) and (47). The controller for the case of (22) and (24) can be obtained with the same way, which will not be considered. The algorithm now proceeds as follows. 


\section{PS Algorithm}

Step 1 (design the parameter matrices). For each subsystem, design a symmetrical polynomial parameter matrix $K_{i}\left(x, k_{m}\right)$ with the terms $K_{i}^{n_{r} n_{c}}=\sum_{s=0}^{\tilde{r}}\left\{\theta_{m} k_{m} \prod_{\tilde{j}=1}^{n} x_{\tilde{j}}^{s_{\tilde{j}}}\right\}$, where $\theta_{m}$ is the coefficient of the item, $k_{m}$ is the parameter, $m$ is the serial number of the parameter, $s$ is the serial number of the monomial with maximum number $\widetilde{r}, n_{r}$ is the row number and $n_{c}$ is the column number for the term in matrix $K_{i}\left(x, k_{m}\right)$, $s_{\tilde{j}}$ is a nonnegative integer, and $\tilde{j}$ is the serial number of the state variable with the maximum number $n$.

Step 2 (obtain inequality conditions).

(2.1) Obtain the values of parameters: choose some symmetric positive definite matrices $P_{i}$ such that (23) holds. Then, let $a_{1}=(1 / 2) \min _{i \in M} \lambda_{m}\left(P_{i}\right)$, $a_{2}=(1 / 2) \max _{i \in M} \lambda_{M}\left(P_{i}\right), a_{3}=\max _{i \in M}\left\|P_{i}\right\|=$ $\max _{i \in M}\left(\sum_{j=1}^{n} \sum_{i=1}^{n} P(i, j)^{2}\right)^{1 / 2}, \mu=\max _{k, l \in M}\left(\lambda_{M}\left(P_{k}\right) /\right.$ $\left.\lambda_{m}\left(P_{l}\right)\right)$. Obtain $\kappa, \eta$ according to Assumption 12 .

(2.2) Obtain inequality conditions: with the given average dwell time $\tau_{\alpha}$ and the obtained parameter values, achieve inequality (31), and express it with the form of $f_{i 0}>0$. Substituting $K_{i}\left(x, k_{m}\right)$ into (47) leads to the inequality conditions $G_{i}>0$.

Step 3 (simplify the inequality conditions).

(3.1) Considering the polynomial expressions $G_{i}\left(x, k_{m}, \alpha_{i}\right)$ for the $i$ th subsystem, set the coefficient of the terms, whose degree subjects to the system state $x$ are odd to be zero, and obtain a group of equalities. Transform the equalities to express one parameter with other parameters as follows: $k_{m_{s}}=f\left(\alpha_{i}, k_{m_{j_{1}}}\right.$, $\left.k_{m_{j_{2}}}, \ldots\right), \forall m_{s}, m_{j_{1}}, m_{j_{2}} \in m, m_{s} \neq m_{j_{1}}, m_{s} \neq m_{j_{2}}$. Then, substitute $f\left(\alpha_{i}, k_{m_{j_{1}}}, k_{m_{j_{2}}}, \ldots\right)$ with $k_{m_{s}}$ in $K_{i}$.

(3.2) Choose some terms in the polynomial expressions $G_{i}\left(x, k_{m}, \alpha_{i}\right)$ and set their coefficients to be zero. Then, a group of equalities are obtained. Transform the equalities to express one parameter with other parameters as the forms $k_{m_{r}}=f\left(\alpha_{i}, k_{m_{k_{1}}}\right.$, $\left.k_{m_{k_{2}}}, \ldots\right), \forall m_{r}, m_{k_{1}}, m_{k_{2}} \in m, m_{r} \neq m_{k_{1}}, m_{r} \neq m_{k_{2}}$. Substitute $f\left(\alpha_{i}, k_{m_{k_{1}}}, k_{m_{k_{2}}}, \ldots\right)$ with $k_{m_{r}}$ of $K_{i}$. Repeat this process several times until only one parameter $k_{m}$ retained in each parameter matrix $K_{i}$.

(3.3) Obtain a new polynomial inequality $G_{i}>0$ with the new $K_{i}\left(x, k_{i}\right)$ and rewrite it into quadratic form. Then the positive of the quadratic form is equivalent to the positive definiteness of quadratic matrix $\widehat{P}_{i}\left(k_{i}, \alpha_{i}\right)$.

(3.4) According to the positive definiteness of the quadratic matrix $\widehat{P}_{i}\left(k_{i}, \alpha_{i}\right)$, we know that all the order principal minors of the quadratic form matrix are positive that are $f_{i r}>0, r \in N_{+}$. Besides, we know that $f_{i 0}>0$. In this way, a polynomial inequality constraint condition set $C_{i}$, which is an equivalent condition of (31) and (47), is obtained.
(3.5) Repeat (3.1)-(3.4) and obtain the polynomial inequality constraint condition set $C_{i}$ for each subsystem.

Step 4 (solve the interval values of the parameters).

(4.1) Regard every inequality in $C_{i}$ as implicit function, such as $f_{i r}\left(k_{i}, \alpha_{i}\right)=0$ and $f_{i 0}\left(\alpha_{i}\right)=0$, and draw them in the $k_{i}-\alpha_{i}$ plane. Then, the $k_{i}, \alpha_{i}$ parameter space is decomposed into many cells.

(4.2) Search out the required region $\mathrm{RC}_{i}$ by verifying any one point in each cell to all the constraint conditions in $C_{i}$.

(4.3) Repeat (4.1)-(4.2) for each subsystem and obtain the required region $\mathrm{RC}_{i}, i \in M$ for each subsystem.

Step 5 (obtain controllers).

(5.1) For every subsystem, achieve the parameterized controller by substituting the new $K_{i}\left(x, k_{i}\right)$ obtained from Step (3.2) into (25).

(5.2) According to the required regions $\mathrm{RC}_{i}$ obtained in Step (4.2), choose several values for each $k_{i}$ and substitute them into the parameterized controller. Then, several group controllers are obtained.

(5.3) Simulate system (1) with every group controllers obtained in Step (5.2), respectively. Compare the simulating results and find out the controller possessing better capability.

Remark 16. In Step (3.3), $G_{i}$ is updated with the new $K_{i}\left(x, k_{i}\right)$. Then, the new $G_{i}$ is transformed into quadratic form. If infeasible result is got, redesign the parameter matrix $K_{i}\left(x, k_{m}\right)$ again and rerun the algorithm.

Remark 17. By Step (4.2), for each subsystem, the parameter space is decomposed into cells. According to the Cylindrical Algebraic Decomposition algorithm [31], all the points containing in each cell have the same characteristics. Then, in Step (4.2), at least one required region $\mathrm{RC}_{i}$ satisfying the requirements of $C_{i}$ is obtained by verifying any one point in each cell. If infeasible result is got in Step (4.2), redesign the corresponding parameter matrix $K_{i}\left(x, k_{m}\right)$ and rerun this algorithm for the corresponding subsystem.

\section{Example and Simulations}

In order to verify the effectiveness of the method produced in this paper for the uncertain SPNSs with external disturbance, an example is simulated.

Example 18. Consider the SPNS consisting of two subsystems with following parameters:

$$
f_{1}(x)=\left[\begin{array}{c}
-8 x_{2}-2 x_{2} x_{3}-4.5 x_{1} \\
2 x_{1}-6 x_{2} \\
2 x_{1} x_{2}-4.5 x_{3}
\end{array}\right],
$$




$$
\begin{aligned}
& f_{2}(x)=\left[\begin{array}{c}
1.5 x_{1}+6 x_{2} \\
-3 x_{1}+2 x_{2}^{2} x_{3}+x_{2} \\
-2 x_{2}^{3}+x_{3}
\end{array}\right] \\
& \Delta f_{1}(x)=\left[\begin{array}{c}
0 \\
\theta_{1} e^{-t} x_{1} \\
0
\end{array}\right] \\
& \Delta f_{2}(x)=\left[\begin{array}{c}
0 \\
\theta_{2} x_{1} \\
\theta_{3} x_{2}
\end{array}\right] \\
& g_{1}(x)=\left[\begin{array}{lll}
0 & 2 & 0.5 \\
2 & 1 & 1 \\
0 & 1 & 1
\end{array}\right] \\
& g_{2}(x)=\left[\begin{array}{lll}
0 & 1 & 3 \\
3 & 1 & 2 \\
0 & 2 & 1
\end{array}\right] \\
& h_{1}(x)=\left[\begin{array}{c}
8 x_{2} \\
2 x_{1}+4 x_{2}+x_{3} \\
0.5 x_{1}+4 x_{2}+x_{3}
\end{array}\right] \text {, } \\
& h_{2}(x)=\left[\begin{array}{c}
6 x_{2} \\
x_{1}+2 x_{2}+4 x_{3} \\
3 x_{1}+4 x_{2}+2 x_{3}
\end{array}\right] \text {, } \\
& g_{11}(x)=\operatorname{diag}(1,1,1), \\
& g_{21}(x)=\operatorname{diag}(1,1,1), \\
& \tau_{\alpha}=1 \\
& \gamma=6
\end{aligned}
$$

where $\theta_{1} \in[-1,1], \theta_{2} \in[-0.1,0.1], \theta_{3} \in[-0.2,0.2]$.

4.1. Solve Example 18 with the Method of This Paper. Let $M=$ $\{1,2\}, P_{1}=\operatorname{diag}(1,4,1)$, and $P_{2}=\operatorname{diag}(1,2,2)$. Design the following piecewise Lyapunov function:

$$
V(x)=V_{\sigma(t)}(x)=\frac{1}{2} x^{T} P_{\sigma(t)} x .
$$

Considering the parameter uncertainty, we can get $\left\|\Delta f_{1}(t, x)\right\| \leq\left|\theta_{1}\right| e^{-t}\|x\|,\left\|\Delta f_{2}(t, x)\right\| \leq \theta\|x\|, \theta \in$ $\max \left\{\left|\theta_{2}, \theta_{3}\right|\right\}$. Thus, $\int_{t_{0}}^{t} \varsigma(\tau) d \tau \leq \int_{t_{0}}^{t}\left(\theta+\left|\theta_{1}\right| e^{-t}\right) d \tau \leq \kappa(t-$ $\left.t_{0}\right)+\eta$, where $\kappa=0.2, \eta=1$.

To be sure, $i \in M=\{1,2\}$, which is the same in hereinafter. It is easy to verify that (23) holds with $r_{i}=$ $\operatorname{diag}(1,1,1)$. With the way of Step 2 of the PS algorithm, it gives $a_{1}=1 / 2, a_{2}=2, a_{3}=\sqrt{18}, \mu=4$, which render that (5), (6), and (30) hold.

In the following subsection, the parameterized controllers will be designed and the interval value of their parameters will be solved with the PS algorithm described in Section 3.2.

By $P_{1}$ and $P_{2}$, each subsystem can be transformed into the form of (12), where

$$
\begin{aligned}
& J_{1}(x)=\left[\begin{array}{ccc}
0 & -2 & -2 x_{2} \\
2 & 0 & 0 \\
2 x_{2} & 0 & 0
\end{array}\right], \\
& R_{1}(x)=\left[\begin{array}{ccc}
\frac{9}{2} & 0 & 0 \\
0 & \frac{3}{2} & 0 \\
0 & 0 & \frac{9}{2}
\end{array}\right] \\
& J_{2}(x)=\left[\begin{array}{ccc}
0 & 3 & 0 \\
-3 & 0 & x_{2}^{2} \\
0 & -x_{2}^{2} & 0
\end{array}\right], \\
& R_{2}(x)=\left[\begin{array}{rrc}
-\frac{3}{2} & 0 & 0 \\
0 & -\frac{1}{2} & 0 \\
0 & 0 & -\frac{1}{2}
\end{array}\right] .
\end{aligned}
$$

By substituting the values of all the parameters into (31), the conditions $f_{10}\left(\alpha_{1}\right)>0$ (see $\left.(54)\right)$ and $f_{20}\left(\alpha_{2}\right)>0$ (see (55)) are obtained.

By Step 1 of the PS algorithm, define the following parameter matrix:

$$
K_{i}\left(x, k_{m}\right)=\operatorname{diag}\left(k_{i}, k_{i}, k_{i}\right) .
$$

Then, by (25) and (51), the parameterized controllers for the subsystems of Example 18 are obtained as

$$
\begin{aligned}
& u_{1}\left(k_{1}, x\right)=\left[\begin{array}{c}
-8\left(k_{1}+1\right) x_{2} \\
-\left(k_{1}+1\right)\left(2 x_{1}+4 x_{2}+x_{3}\right) \\
-\left(k_{1}+1\right)\left(0.5 x_{1}+4 x_{2}+x_{3}\right)
\end{array}\right], \\
& u_{2}\left(k_{2}, x\right)=\left[\begin{array}{c}
-6\left(k_{2}+1\right) x_{2} \\
-\left(k_{2}+1\right)\left(x_{1}+2 x_{2}+4 x_{3}\right) \\
-\left(k_{2}+1\right)\left(3 x_{1}+4 x_{2}+2 x_{3}\right)
\end{array}\right] .
\end{aligned}
$$

Hence, by the values of $\gamma, R_{1}(x), R_{2}(x)$, and (51), with the way of Steps 2 and 3, we have 


$$
\begin{aligned}
& \widehat{P}_{1}=\left[\begin{array}{crc}
x_{2}^{2}+\frac{17}{4} k_{1}+\frac{647}{144}-\frac{1}{2} \alpha_{1} & 10 k_{1} & \frac{5}{2} k_{1} \\
* & 96 k_{1}+\frac{215}{9}-2 \alpha_{1} & 8 k_{1} \\
* & * & 2 k_{1}+\frac{647}{144}-\frac{1}{2} \alpha_{1}
\end{array}\right], \\
& \widehat{P}_{2}=\left[\begin{array}{ccc}
10 k_{2}-\frac{217}{144}-\frac{1}{2} \alpha_{2} & 14 k_{2} & 10 k_{2} \\
* & 56 k_{2}-\frac{73}{36}-\alpha_{2} & 16 k_{2} \\
* & * & 20 k_{2}-\frac{73}{36}-\alpha_{2}
\end{array}\right]
\end{aligned}
$$

where “*” means the symmetric terms in the symmetric matrix $\widehat{P}_{i}$.
According to the symmetric positive definiteness of $\widehat{P}_{i}$, the constraint conditions $f_{\text {is }}>0, s \in\{1,2,3\}$ are obtained with the way of Step (3.4) of the PS algorithm.

$C_{1}$

$$
\begin{aligned}
& =\left\{\begin{array}{l}
f_{10}=\alpha_{1}-11.57>0 \\
f_{11}=\frac{647}{144} k_{1}+\frac{17}{4}-\frac{1}{2} \alpha_{1}>0 \\
f_{12}=\frac{19183}{36} k_{1}-\frac{113}{2} k_{1} \alpha_{1}+\frac{139105}{1296}+308 k_{1}^{2}+\alpha_{1}^{2}-\frac{1507}{72} \alpha_{1}>0 \\
f_{13}=-\frac{170161}{1152} \alpha_{1}+\frac{13524241}{5184} k_{1}+\frac{90000935}{186624}+144 k_{1}^{3}-\frac{1}{2} \alpha_{1}^{3}-\frac{445}{2} k_{1}^{2} \alpha_{1}+\frac{121}{4} \alpha_{1}^{2} k_{1}+\frac{36229}{18} k_{1}^{2}-\frac{161899}{288} k_{1} \alpha_{1}+\frac{359}{24} \alpha_{1}^{2}>0,
\end{array}\right. \\
& C_{2}=\left\{\begin{array}{l}
f_{20}=\alpha_{2}-11.57>0 \\
f_{21}=10 k_{2}-\frac{217}{144}-\frac{1}{2} \alpha_{2}>0 \\
f_{22}=364 k_{2}^{2}-\frac{314}{3} k_{2}-38 k_{2} \alpha_{2}+\frac{15841}{5184}+\frac{121}{48} \alpha_{2}+\frac{1}{2} \alpha_{2}^{2}>0 \\
f_{23}=3600 k_{2}^{3}-\frac{20186}{9} k_{2}^{2}-896 k_{2}^{2} \alpha_{2}+\frac{354269}{1296} k_{2}+\frac{8357}{36} k_{2} \alpha_{2}+48 k_{2} \alpha_{2}^{2}-\frac{1156393}{186624}-\frac{10585}{1296} \alpha_{2}-\frac{509}{144} \alpha_{2}^{2}-\frac{1}{2} \alpha_{2}^{3}>0 .
\end{array}\right.
\end{aligned}
$$

Decompose the $\alpha_{1}-k_{1}$ plane into cells by drawing all the curves of $f_{1 j}=0, j \in\{0,1,2,3\}$ in the plane and search out the required cell $\mathrm{RC}_{1}$ with the method of Step (4.2) of the PS algorithm. The required cell $\mathrm{RC}_{1}$ marked by gray background is surrounded by the curves of $f_{10}=0$ and $f_{13}=0$ in Figure 1. Following the above similar procedures, obtain the $\mathrm{RC}_{2}$ that is partly surrounded by the curves of $f_{20}=0$ and $f_{23}=0$ in Figure 2. Then, considering $\mathrm{RC}_{i}$ and (54) and (55), we know that $k_{1} \in\left[3.61, \widetilde{k}_{1}\right], \widetilde{k}_{1} \geq 15$ and $k_{2} \in\left[2.54, \widetilde{k}_{2}\right], \widetilde{k}_{2} \geq 15$. Choose

$$
\begin{aligned}
& k_{1}=5 \\
& k_{2}=5 .
\end{aligned}
$$
law:

By $\tau_{\alpha}=1$, for $i, j \in M$, design the following switching

$$
\begin{aligned}
& \sigma(t) \\
& = \begin{cases}1, & t \in[2 k+0.2,2 k+1.2), \\
2, & t \in[2 k+1.2,2 k+2.2) \text { or } t \in[0,0.2), k \in N .\end{cases}
\end{aligned}
$$
(52)

Obtain a group of controllers by substituting (56) into

Suppose initial states $x\left(t_{0}\right)=\left[\begin{array}{lll}-4 & 1 & -7\end{array}\right]^{\mathrm{T}}$ and external disturbance $w=0$. Let $\theta_{1}=\cos (t), \theta_{2}=0.1 \sin (t)$, and $\theta_{3}=0.2 \sin (t+1)$. Simulate Example 18 under switching law (57) and parameterized controller (52) with parameter 


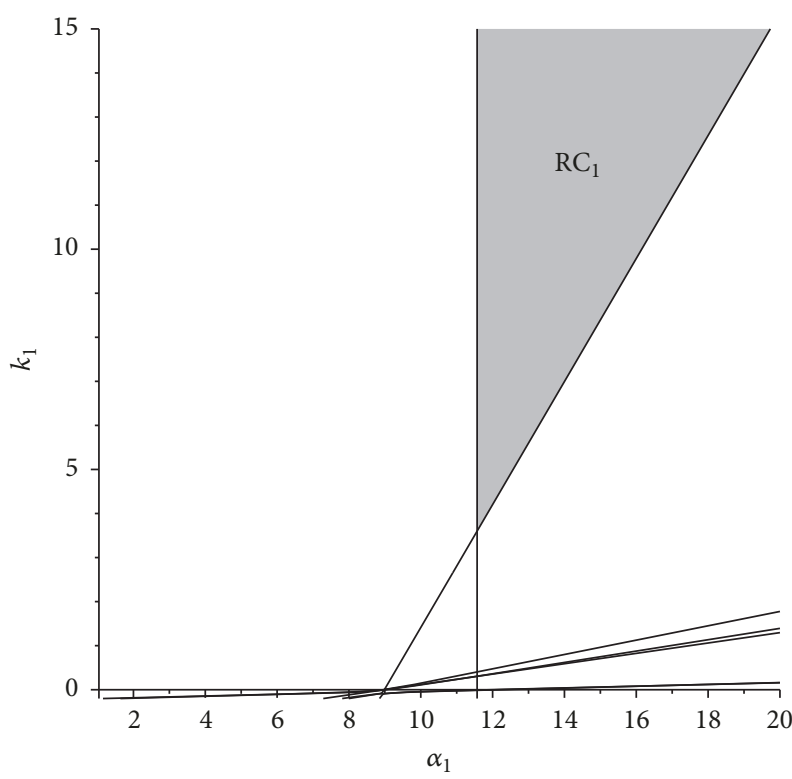

FIGURE 1: System constraint curves in $k_{1}-\alpha_{1}$ plane.

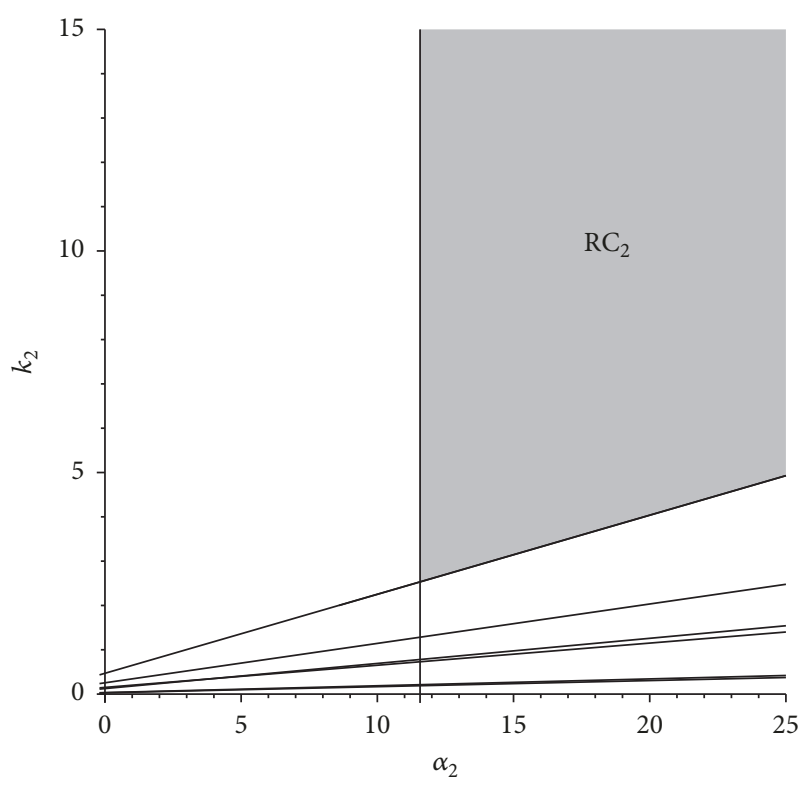

FIGURE 2: System constraint curves in $k_{2}-\alpha_{2}$ plane.

(56). Figure 3 (dotted lines) shows the state responses and Figure 4 (dotted lines) shows the partial enlargement of the state responses.

When $x\left(t_{0}\right)=\left[\begin{array}{lll}0 & 0 & 0\end{array}\right]^{\mathrm{T}}$, suppose external disturbance $w=\left[\begin{array}{lll}e^{-t} & e^{-t} & e^{-t}\end{array}\right]^{\mathrm{T}}$ is inserted into Example 18. To clearly see the disturbance attenuation capabilities of the controllers designed with the method of this paper, define the function

$$
z(t)
$$

$$
=\frac{\int_{t_{0}}^{t} \exp \left(\left(-(\ln (\mu \xi)) / \tau_{\alpha}\right)\left(\tau-t_{0}\right)\right) y^{T}(\tau) y(\tau) d \tau}{\int_{t_{0}}^{t} w^{T}(\tau) w(\tau) d \tau}
$$
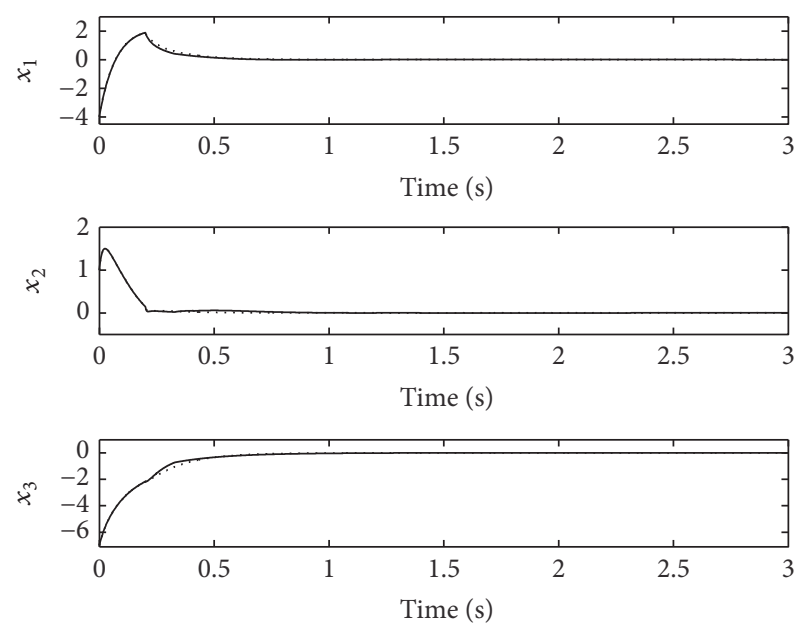

Case 1

- Method in [22]

FIgURE 3: State response of case 1 (dotted lines) and the method of [22] (solid lines).
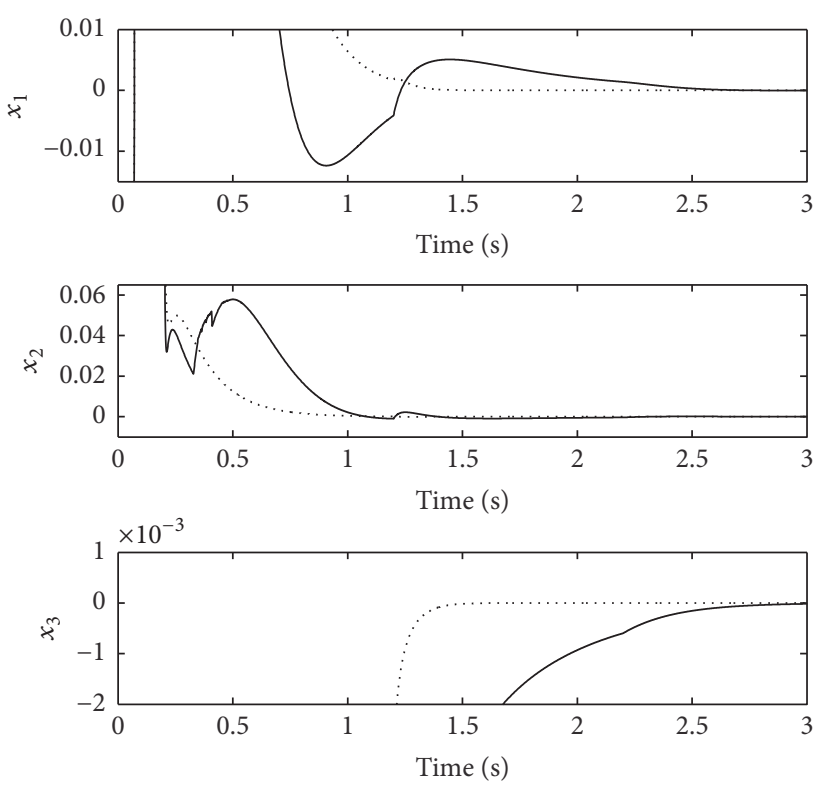

.... Case 1

- Method in [22]

Figure 4: Partial enlargement of the state response.

to describe the $L_{2}$ disturbance attenuation gain level of the system from $w(t)$ to $y(t)$. Simulate the system with the switching law (57) and the parameterized controller (52) with parameter (56). The simulation results are showed by Figure 5 (dotted line). In order to verify the tuning characteristic of the parameter containing in the parameterized controller, choose

$$
\begin{aligned}
& k_{1}=10 \\
& k_{2}=10,
\end{aligned}
$$




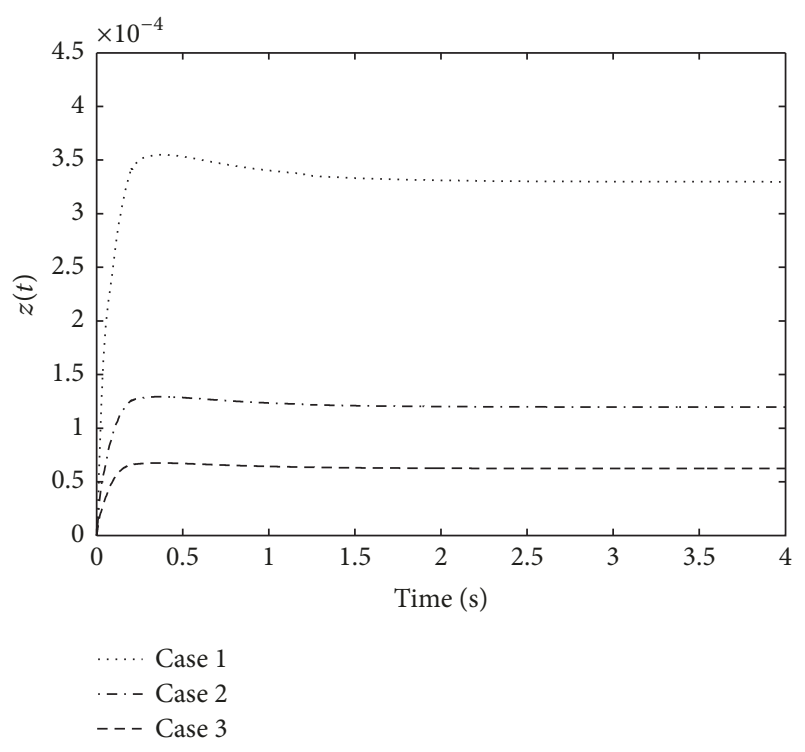

FIGURE 5: The curves of $z(t)$ with different parameter values.

$$
\begin{aligned}
& k_{1}=15 \\
& k_{2}=15
\end{aligned}
$$

respectively, and identify them as cases 2 and 3, respectively. Meanwhile, identify the previous case as case 1 . Simulate the example of case 2 and case 3, respectively, again. The curves of $z(t)$ are showed, respectively, with dot and dash line (case 2 ) and dash line (case 3 ) in Figure 5.

Summary 1. According to Figure 5, we know that the disturbance attenuation capability of the parameterized controller is more and more bigger as the values of the parameters increase, which means that the optimized controller can be obtained by choosing larger values for the parameter. The simulation result is in excellent agreement with Remark 15. In other words, when choosing the parameter values according to $\mathrm{RC}_{i}$, the three aims of the parameterized controller described in Remarks 11 and 14 are achieved. When choosing larger parameter values according to $\mathrm{RC}_{i}$ for the parameterized controller (52), Example 18 has better $L_{2}$ gain performance from $w$ to $y$.

4.2. Solve Example 18 with the Method of [22]. In order to verify the effectiveness of the method of Theorem 13, Example 18 is simulated with the method of Theorem 2 of [22]. At first, it will be verified that the method of [22] is suitable for Example 18.

As with the method of this paper, choose the piecewise Lyapunov function $V(x)=V_{\sigma(t)}(x)$ as (49), where $V_{1}(x)=$ $0.5 x_{1}+2 x_{2}+0.5 x_{3}$ and $V_{1}(x)=0.5 x_{1}+x_{2}+x_{3}$. It is easy to verify that the 1th subsystem is inherently passive and the 2 th subsystem is inherently nonpassive with $L_{f_{2}} V_{2}(x) \leq$ $3 V_{2}(x)$. Besides, considering $g_{11}(x)=\operatorname{diag}(1,1,1), g_{21}(x)=$ $\operatorname{diag}(1,1,1)$, we know that Example 18 has the structure of system (1) of [22].

On the other hand, by $V_{i}(x)$, it is easy to obtain the values of the parameters that is $a_{1}=0.5, a_{2}=2, a_{3}=\sqrt{18}, \mu=$ $4, \kappa=0.2, \eta=1, \lambda=3$ and verify that conditions (12)-(16) for Theorem 2 of [22] hold. Noticing that $\dot{V}_{1}(x)=$ $\left(\partial V_{1}(x) / \partial x\right) f_{1}(x) \leq x^{T}\left[\begin{array}{ccc}-4.5 & 0 & 0 \\ 0 & -24 & 0 \\ 0 & 0 & -4.5\end{array}\right] x \leq-9 V_{1}(x)$, by (12) of [22], it gives that $\|x(t+\tau)\| \leq 2 e^{-(9 / 2) \tau}\|x(t)\|$, which means $c=2$ and $\bar{\lambda}=9 / 2$. For $\delta=0.5, \tau=0.0006$, it is easy to verify that the 1th subsystem is exponentially small-time norm-observable with degree $\bar{\lambda}-c \kappa=4.1$. Then, it is easy to verify that $a_{2} c^{2} \leq\left(a_{1}-k_{0}\right) \exp \left(a_{3} \eta / a_{1}\right)$ and $\| h_{1}(x(t+$ $s))\left\|^{2} \leq\left(k_{0} / \tau\right) \exp (-2 \bar{\lambda} \tau)\right\| x(t) \|^{2}$ for any $s \in[0,0.0006]$, where $k_{0}=0.01$ and $t$ satisfies $\left\|h_{1}(x(t+s))\right\| \leq 0.5$ for all $s \in[0,0.0006]$. Let the average dwell time $\tau_{a}=1$ and passivity rate $r=0.5$, the disturbance attenuation level $\gamma=6$ and $\lambda_{2}=0.05$. Then, $\lambda_{1}=\lambda_{2}+a_{3}^{2} / 4 a_{1} \gamma^{2}=0.3, \lambda^{*}=$ $\lambda_{1} / r+\lambda / r+(\ln \mu) / r \tau_{a}+a_{3} \kappa / r a_{1}-\lambda=9.7667$ and $k=$ $\bar{\lambda}-c \kappa-(1 / 2)\left(\lambda^{*}-a_{3} \kappa / a_{1}-a_{3}^{2} / 4 a_{1} \gamma^{2}\right)=0.1902>0$. By the obtained parameters, design controller (61)

$$
\begin{aligned}
& u_{1}(x)=\left\{\begin{array}{ll}
{\left[\frac{4.8348\left(x_{1}^{2}+4 x_{2}^{2}+x_{3}^{2}\right)}{L(x)}+1\right]\left[\begin{array}{c}
-8 x_{2} \\
-2 x_{1}-4 x_{2}-x_{3} \\
0
\end{array} \quad L(x)>0.25\right.} \\
-0.5 x_{1}-4 x_{2}-x_{3}
\end{array}\right] \quad L(x) \leq 0.25, \\
& u_{2}(x)=\left[\begin{array}{c}
-6 x_{2} \\
-x_{1}-2 x_{2}-4 x_{3} \\
-3 x_{1}-4 x_{2}-2 x_{3}
\end{array}\right],
\end{aligned}
$$

where $L(x)=64 x_{2}^{2}+\left(2 x_{1}+4 x_{2}+x_{3}\right)^{2}+\left(0.5 x_{1}+4 x_{2}+x_{3}\right)^{2}$.

Then, according to Theorem 2 of [22], under controller (61) and switching law (57), the system of Example 18 is globally robustly exponentially stable when $w \equiv 0$ and has $L_{2}$ gain disturbance attenuation level $\gamma$ from $w$ to $y$.
Summary 2. Comparing between (52) and (61), we know that the controllers designed with the method of this paper is simpler than that designed with the method of [22].

Let $\theta_{1}=\cos (t), \theta_{2}=0.1 \sin (t), \theta_{3}=0.2 \sin (t+1)$. With the same initial states $x\left(t_{0}\right)=\left[\begin{array}{lll}-4 & 1 & -7\end{array}\right]^{\mathrm{T}}$, simulate Example 18 


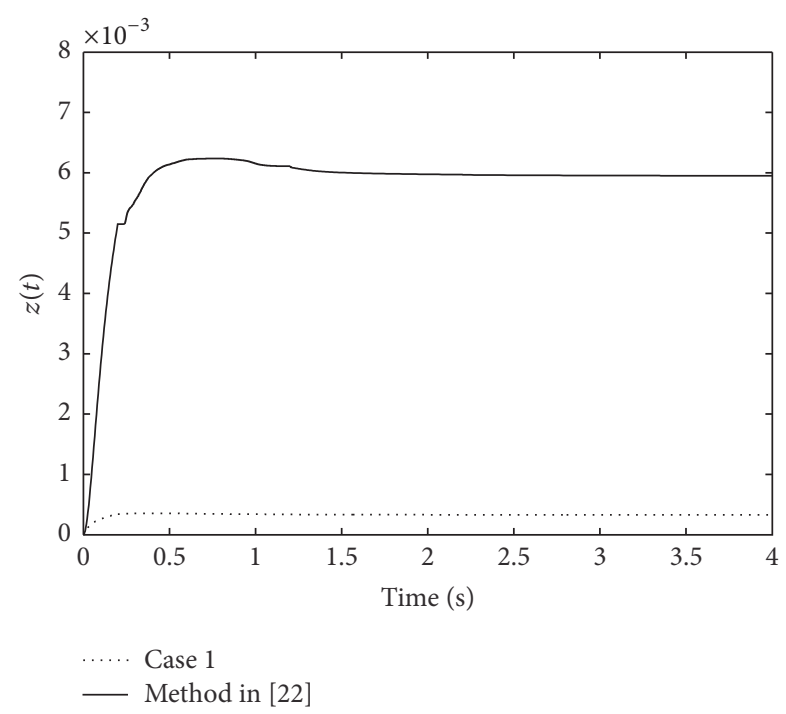

Figure 6: The curves of $z(t)$ with the method of this paper in case 1 and [22].

under controller (61) and switching law (57). Figure 3 (solid lines) shows the state responses and Figure 4 (solid lines) shows the partial enlargement of the state responses.

Summary 3. According to Figures 3 and 4, the system controlled with the method of this paper became stable for no more than $1.5 \mathrm{~s}$ (see the dotted lines in Figures 3 and 4), while the system controlled with the method of [22] became stable for over $2.5 \mathrm{~s}$ (see the solid lines in Figures 3 and 4). Overall, the controller designed using Theorem 13 of this paper possesses a better robustness compared with the existing approach [22].

When $x\left(t_{0}\right)=\left[\begin{array}{lll}0 & 0 & 0\end{array}\right]^{\mathrm{T}}$, suppose external disturbance $w=\left[\begin{array}{lll}e^{-t} & e^{-t} & e^{-t}\end{array}\right]^{\mathrm{T}}$ is inserted into Example 18 under controller (61) and switching law (57). Example 18 is simulated and the disturbance attenuation $L_{2}$ gain from $w$ to $y$ is showed by Figure 6 (solid line).

Summary 4. With the same external disturbance and $L_{2}$ gain disturbance attenuation level $\gamma$, Figure 6 illustrates that the controllers designed using Theorem 13 of this paper provide better disturbance attenuation capability compared with the controller using the existing approach described in [22].

\section{Conclusions}

In this paper, the exponential feedback passivity of switched polynomial nonlinear systems is presented. Comparing with the existing results of [25-27], the nonlinear functions of the systems do not need to satisfy the so-called Lipschitz condition. In addition, the performance of the SPNSs can be improved by adjusting the value of the parameter contained in the parameterized controller according to the obtained feasible interval solution of the parameters.

\section{Conflicts of Interest}

The authors declare that they have no conflicts of interest.

\section{Acknowledgments}

This work is supported by the National Natural Science Foundation of China under Grant 61374001, Scientific Research Fund of Sichuan Provincial Education Department under Grants 14ZB0318, and Scientific Research Fund of Sichuan University of Arts and Science under Grant 2016KZ003Y.

\section{References}

[1] Z. Li, Y. Qiao, H. Qi, and D. Cheng, "Stability of switched polynomial systems," Journal of Systems Science and Complexity, vol. 21, no. 3, pp. 362-377, 2008.

[2] J. Xu, L. Xie, and Y. Wang, "Simultaneous stabilization and robust control of polynomial nonlinear systems using SOS techniques," Institute of Electrical and Electronics Engineers Transactions on Automatic Control, vol. 54, no. 8, pp. 1892-1897, 2009.

[3] G.-R. Yu, Y.-C. Huang, and C.-Y. Cheng, "Robust Ho controller design for polynomial fuzzy control systems by sum-ofsquares approach," IET Control Theory \& Applications, vol. 10, no. 14, pp. 1684-1695, 2016.

[4] M. Wang and J. Zhao, "Quadratic stabilization of a class of switched nonlinear systems via single Lyapunov function," Nonlinear Analysis: Hybrid Systems, vol. 4, no. 1, pp. 44-53, 2010.

[5] M. S. Branicky, "Multiple Lyapunov functions and other analysis tools for switched and hybrid systems," Institute of Electrical and Electronics Engineers Transactions on Automatic Control, vol. 43, no. 4, pp. 475-482, 1998.

[6] B. Niu and J. Zhao, "Robust Ho control for a class of uncertain nonlinear switched systems with average dwell time," International Journal of Control, vol. 86, no. 6, pp. 1107-1117, 2013.

[7] J. Zhao and D. J. Hill, "On stability, Ho control for switched systems," Automatica, vol. 44, no. 5, pp. 1220-1232, 2008.

[8] L. J. Long and J. Zhao, "control of switched nonlinear systems in p-normal form using multiple Lyapunov functions," IEEE Transactions on Automatic Control, vol. 57, no. 5, pp. 1258-1291, 2012.

[9] J. C. Willems, "Dissipative dynamical systems. I. General theory," Archive for Rational Mechanics and Analysis, vol. 45, pp. 321-351, 1972.

[10] D. Hill and P. Moylan, "The Stability of Nonlinear Dissipative Systems," IEEE Transactions on Automatic Control, vol. 21, no. 5, pp. 708-711, 1976.

[11] A. J. Van der Schaft, L2-gain and passivity techniques in nonlinear control, Springer, London, UK, 2nd edition, 1999.

[12] A. Astolfi, R. Ortega, and R. Sepulchre, "Stabilization and disturbance attenuation of nonlinear systems using dissipativity theory," European Journal of Control, vol. 8, no. 5, pp. 408-431, 2002.

[13] Y. I. Son, J. W. Yang, N. H. Jo, H. Shim, and J. H. Seo, "Feedback passivity approach to output feedback disturbance attenuation for uncertain nonlinear systems," International Journal of Systems Science, vol. 35, no. 8, pp. 467-477, 2004.

[14] Z.-P. Jiang, D. J. Hill, and A. . Fradkov, "A passification approach to adaptive nonlinear stabilization," Systems \& Control Letters, vol. 28, no. 2, pp. 73-84, 1996. 
[15] C. I. Byrnes, A. Isidori, and J. C. Willems, "Passivity, feedback equivalence, and the global stabilization of minimum phase nonlinear systems," Institute of Electrical and Electronics Engineers Transactions on Automatic Control, vol. 36, no. 11, pp. 1217, 1228-1240, 1991.

[16] Z. Lin, J. Liu, and Y. Niu, "Robust passivity and feedback design for nonlinear stochastic systems with structural uncertainty," Mathematical Problems in Engineering, vol. 2013, Article ID 460348, 9 pages, 2013.

[17] Y. Liu, G. S. Stojanovski, M. J. Stankovski, G. M. Dimirovski, and J. Zhao, "Feedback passivation of switched nonlinear systems using storage-like functions," International Journal of Control, Automation, and Systems, vol. 9, no. 5, pp. 980-986, 2011.

[18] Y. Wang, V. Gupta, and P. J. Antsaklis, "On passivity of a class of discrete-time switched nonlinear systems," Institute of Electrical and Electronics Engineers Transactions on Automatic Control, vol. 59, no. 3, pp. 692-702, 2014.

[19] H. Pang and J. Zhao, "Robust passivity, feedback passification and global robust stabilisation for switched non-linear systems with structural uncertainty," IET Control Theory \& Applications, vol. 9, no. 11, pp. 1723-1730, 2015.

[20] H. Pang and J. Zhao, "Adaptive feedback passivity-based disturbance attenuation for switched nonlinearly parameterized systems," Transactions of the Institute of Measurement and Control, pp. 1-10, 2016.

[21] Y. Liu and J. Zhao, "Stabilization of switched nonlinear systems with passive and non-passive subsystems," Nonlinear Dynamics, vol. 67, no. 3, pp. 1709-1716, 2012.

[22] C. Li and J. Zhao, "Robust passivity-based $\mathrm{H}_{\infty}$ control for uncertain switched nonlinear systems," International Journal of Robust and Nonlinear Control, vol. 26, no. 14, pp. 3186-3206, 2016.

[23] A. L. Fradkov and D. J. Hill, "Exponential feedback passivity and stabilizability of nonlinear systems," Automatica, vol. 34, no. 6, pp. 697-703, 1998.

[24] V. Chellaboina and W. M. Haddad, "Exponentially dissipative nonlinear dynamical systems: a nonlinear extension of strict positive realness," Mathematical Problems in Engineering, no. 12, pp. 25-45, 2003.

[25] H. Wu, X. Han, L. Wang, Y. Wang, and B. Fang, "Exponential passivity of memristive neural networks with mixed timevarying delays," Journal of The Franklin Institute, vol. 353, no. 3, pp. 688-712, 2016.

[26] S. Senthilraj, R. Raja, Q. X. Zhu, R. Samidurai, and Z. S. Yao, "Exponential passivity analysis of stochastic neural networks with leakage, distributed delays and Markovian jumping parameters," Neurocomputing, vol. 175, pp. 401-410, 2016.

[27] X. Zhang, X. Fan, Y. Xue, Y. Wang, and W. Cai, "Robust exponential passive filtering for uncertain neutral-type neural networks with time-varying mixed delays via Wirtinger-based integral inequality," International Journal of Control, Automation, and Systems, vol. 15, no. 2, pp. 585-594, 2017.

[28] A. Benabdallah, "Exponential passivity for output feedback stabilisation of nonlinear uncertain systems," International Journal of Systems Science, vol. 41, no. 11, pp. 1363-1368, 2010.

[29] J. Hespanha and A. Morse, "Stability of switched systems with average dwell-time," in Proceedings of the 38th IEEE Conference on Decision and Control, pp. 2655-2660, Phoenix, AZ, USA, 1999.

[30] W. Langson and A. Alleyne, "Infinite horizon optimal control of a class of nonlinear systems," in Proceedings of the 1997 American Control Conference, vol. 5, no. 1, pp. 3017-3022, June 1997.
[31] D. S. Armon, G. E. Collins, and S. Mccallum, "Cylindrical algebraic decomposition I: the basic algorithm," Texts and Monographs in Symbolic Computation, vol. 13, no. 4, pp. 878$889,1982$. 


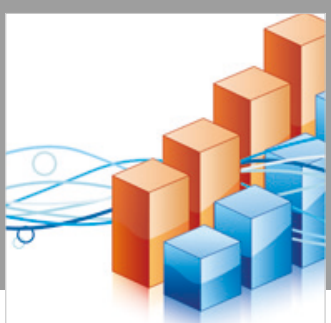

Advances in

Operations Research

\section{-n-m}
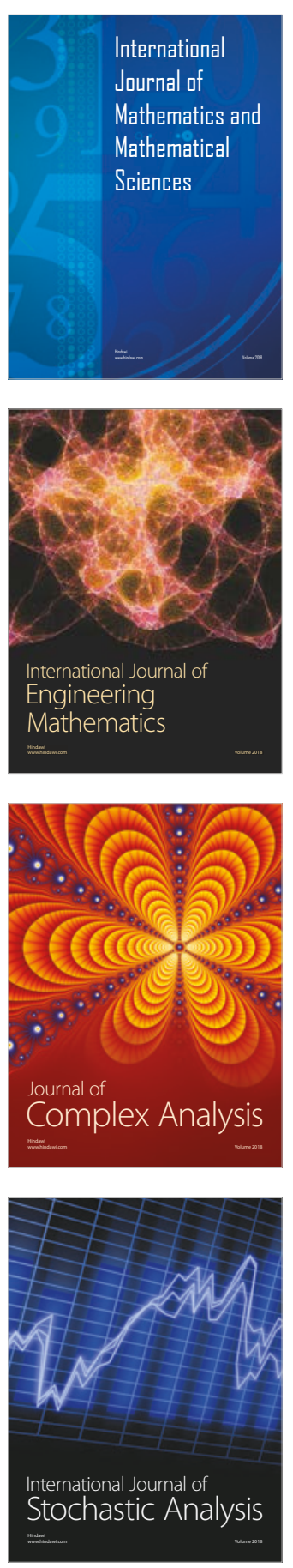
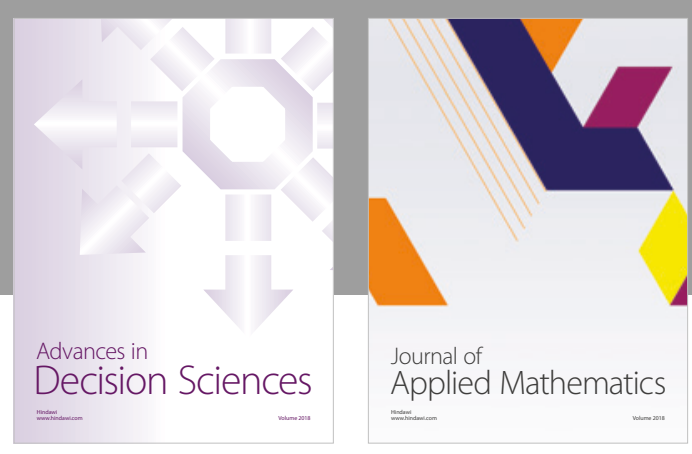

Journal of

Applied Mathematics
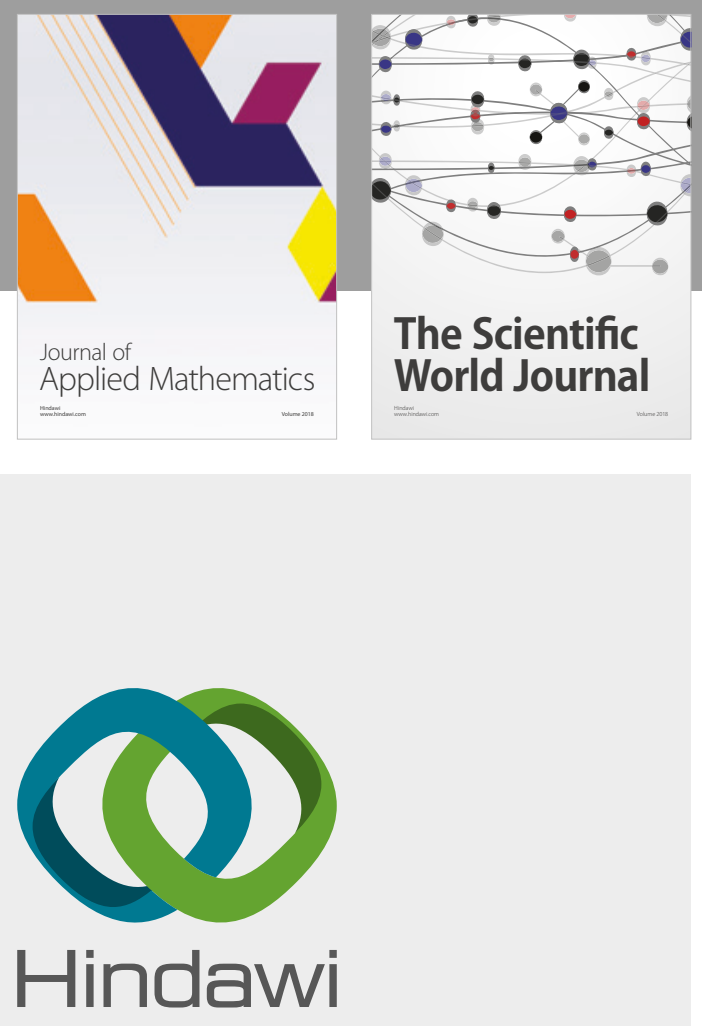

Submit your manuscripts at

www.hindawi.com

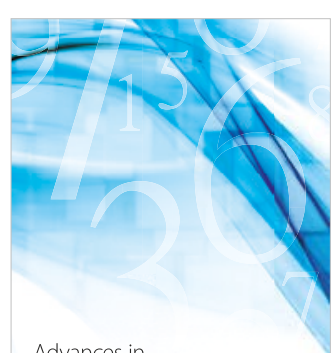

Advances in
Numerical Analysis
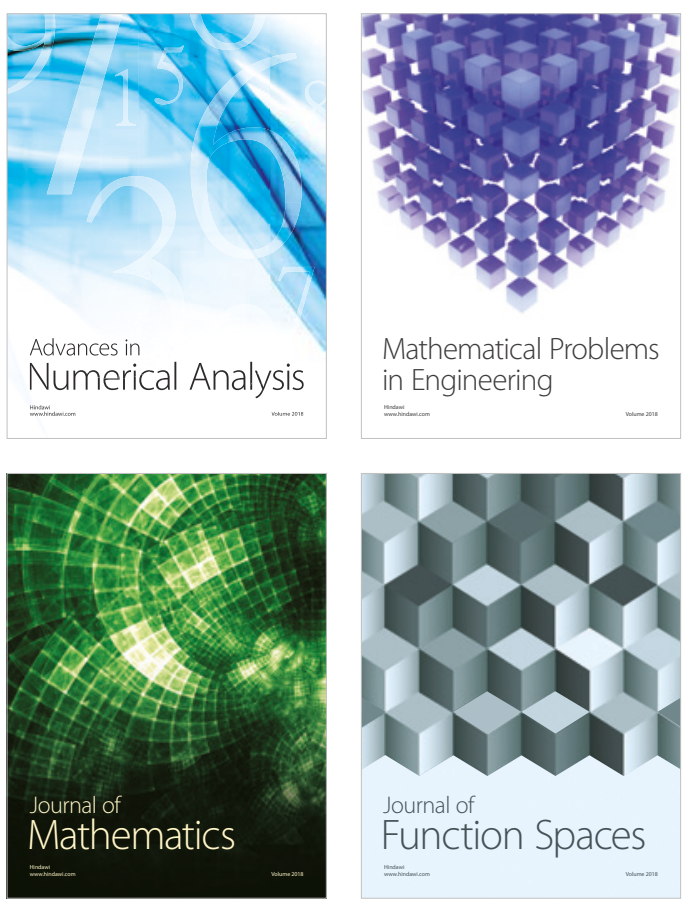

Mathematical Problems in Engineering

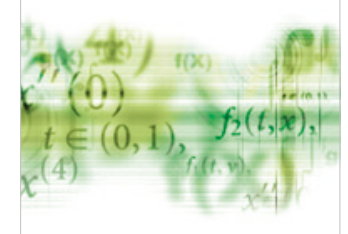

International Journal of

Differential Equations

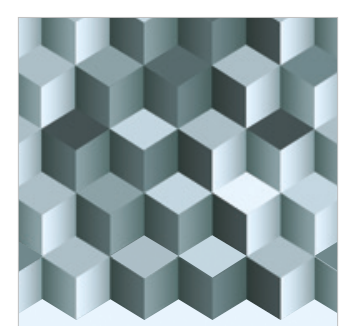

Journal of

Function Spaces

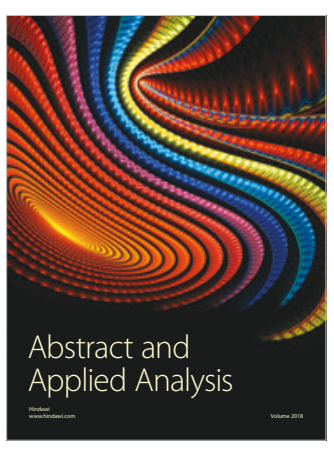

The Scientific

World Journal

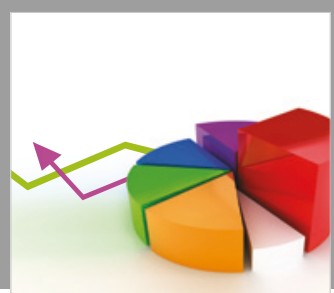

Journal of

Probability and Statistics
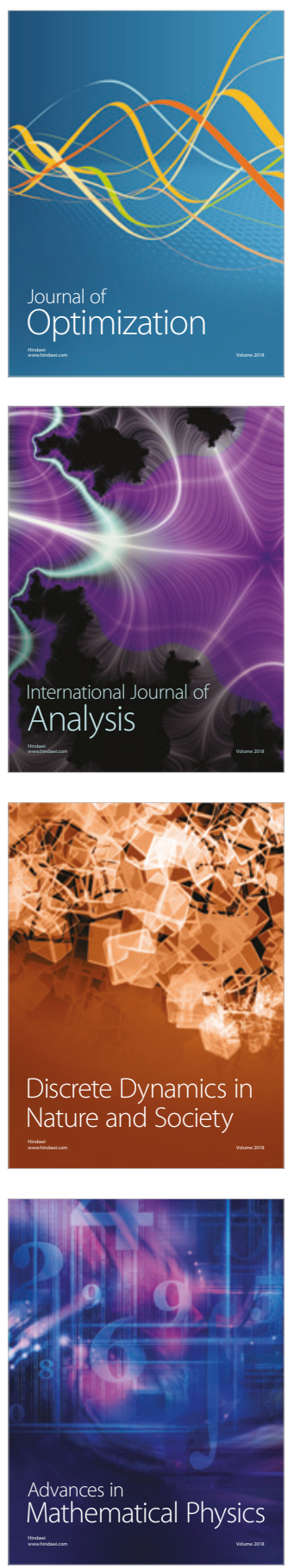